\title{
Algorithmic Stability for Adaptive Data Analysis*
}

\author{
Raef Bassily \\ University of California San \\ Diego, USA \\ Thomas Steinke \\ Harvard University, USA
}

\author{
Kobbi Nissim \\ Ben-Gurion University of the \\ Negev, Israel and Harvard \\ University, USA \\ Uri Stemmer" \\ Ben-Gurion University of the \\ Negev, Israel
}

\author{
Adam Smith \\ Pennsylvania State University, \\ USA \\ Jonathan Ullman** \\ Northeastern University, USA
}

\begin{abstract}
Adaptivity is an important feature of data analysis - the choice of questions to ask about a dataset often depends on previous interactions with the same dataset. However, statistical validity is typically studied in a nonadaptive model, where all questions are specified before the dataset is drawn. Recent work by Dwork et al. (STOC, 2015) and Hardt and Ullman (FOCS, 2014) initiated a general formal study of this problem, and gave the first upper and lower bounds on the achievable generalization error for adaptive data analysis.

Specifically, suppose there is an unknown distribution $\mathbf{P}$ and a set of $n$ independent samples $\mathbf{x}$ is drawn from $\mathbf{P}$. We seek an algorithm that, given $\mathbf{x}$ as input, accurately answers a sequence of adaptively chosen "queries" about the unknown distribution $\mathbf{P}$. How many samples $n$ must we draw from the distribution, as a function of the type of queries, the number of queries, and the desired level of accuracy?

In this work we make two new contributions towards resolving this question:
\end{abstract}

\footnotetext{
${ }^{\dagger}$ Part of this work was done while the author was at Pennsylvania State University, supported by NSF award CDI0941553. rbassily@ucsd.edu

${ }^{\ddagger}$ Supported by a grant from the Sloan Foundation, a Simons Investigator grant to Salil Vadhan, and NSF grant CNS1237235. kobbi@seas . harvard .edu

§Supported by NSF award IIS-1447700, a Google Faculty Award and a Sloan Foundation research award. asmith@psu.edu

`Supported by NSF grants CCF-1116616, CCF-1420938, and CNS-1237235. tsteinke@seas.harvard.edu

"Supported by the Ministry of Science and Technology (Israel), and by the Frankel Center for Computer Science. stemmer@cs.bgu.ac.il

** Part of this work was done while the author was at Columbia University, supported by a Junior Fellowship from the Simons Society of Fellows. jullman@ccs.neu.edu

${ }^{*}$ This work unifies and subsumes the two arXiv manuscripts BSSU15, NS15.
}

Permission to make digital or hard copies of all or part of this work for personal or classroom use is granted without fee provided that copies are not made or distributed for profit or commercial advantage and that copies bear this notice and the full citation on the first page. Copyrights for components of this work owned by others than ACM must be honored. Abstracting with credit is permitted. To copy otherwise, or republish, to post on servers or to redistribute to lists, requires prior specific permission and/or a fee. Request permissions from Permissions@acm.org.

STOC'16, June 19-21, 2016, Cambridge, MA, USA

(c) 2016 ACM. $978-1-4503-4132-5 / 16 / 06 \ldots \$ 15.00$

http://dx.doi.org/10.1145/2897518.2897566
1. We give upper bounds on the number of samples $n$ that are needed to answer statistical queries. The bounds improve and simplify the work of Dwork et al. (STOC, 2015), and have been applied in subsequent work by those authors (Science, 2015; NIPS, 2015).

2. We prove the first upper bounds on the number of samples required to answer more general families of queries. These include arbitrary low-sensitivity queries and an important class of optimization queries (alternatively, risk minimization queries).

As in Dwork et al., our algorithms are based on a connection with algorithmic stability in the form of differential privacy. We extend their work by giving a quantitatively optimal, more general, and simpler proof of their main theorem that the stability notion guaranteed by differential privacy implies low generalization error. We also show that weaker stability guarantees such as bounded KL divergence and total variation distance lead to correspondingly weaker generalization guarantees.

\section{Categories and Subject Descriptors}

G.3 [Probability and Statistics]: Multivariate statistics; H.4 [Information Systems Applications]: Miscellaneous D.2.8 [Software Engineering]: Metrics - complexity measures, performance measures

\section{Keywords}

Adaptivity, Generalization, Data Analysis, Stability, Differential Privacy, Statistics

\section{INTRODUCTION}

Multiple hypothesis testing is a ubiquitous task in empirical research. A finite sample of data is drawn from some unknown population, and several analyses are performed on that sample. The outcome of an analysis is deemed significant if it is unlikely to have occurred by chance alone, and a "false discovery" occurs if the analyst incorrectly declares an outcome to be significant. False discovery has been identified as a substantial problem in the scientific community (see e.g. Ioa05, GL14]). This problem persists despite decades of research by statisticians on methods for preventing false discovery, such as the widely used Bonferroni Correction Bon36, Dun61 and the Benjamini-Hochberg Procedure BH95. 
Some probability of false discovery is unavoidable, but false discoveries appear to be more prevalent in practice than the theory predicts. This high rate of false discovery is often attributed to misuse of statistics. An alternative explanation is that the prevalence of false discovery arises from the inherent adaptivity in the data analysis processthe fact that the choice of analyses to perform depends on previous interactions with the data (see e.g. GL14]). Adaptivity is essentially unavoidable when a sequence of research groups publish research papers based on overlapping data sets. Adaptivity also arises naturally in other settings, for example: in multistage inference algorithms where data are preprocessed (say, to select features or restrict to a principal subspace) before the main analysis is performed; in scoring data-based competitions $\mathrm{BH} 15]$; and in the re-use of holdout or test data $\mathrm{DFH}^{+} 15 \mathrm{c}, \mathrm{DFH}^{+} 15 \mathrm{a}$.

The general problem of adaptive data analysis was formally modeled and studied in recent papers by Dwork, Feldman, Hardt, Pitassi, Reingold, and Roth $\mathrm{DFH}^{+} 15 \mathrm{~b}$ and by Hardt and Ullman HU14. The striking results of Dwork et al. $\mathrm{DFH}^{+} 15 \mathrm{~b}$ gave the first nontrivial algorithms for provably ensuring statistical validity in adaptive data analysis, allowing for even an exponential number of tests against the same sample. In contrast, HU14, SU15b showed inherent statistical and computational barriers to preventing false discovery in adaptive settings.

The key ingredient in Dwork et al. is a notion of "algorithmic stability" that is suitable for adaptive analysis. Informally, changing one input to a stable algorithm does not change it's output "too much." Traditionally, stability was measured via the change in the generalization error of an algorithm's output, and algorithms stable according to such a criterion have long been known to ensure statistical validity in nonadaptive analysis DW79a, DW79b, KR99, BE02, SSSS10. Following a connection first suggested by McSherry ${ }^{1}$ Dwork et al. showed that a stronger stability condition designed to ensure data privacy - called differential privacy (DP) DMNS06, Dwo06-guarantees statistical validity in adaptive data analysis. This allowed them to repurpose known DP algorithms to prevent false discovery. A crucial difference from traditional notions of stability is that DP requires a change in one input lead to a small change in the probability distribution on the outputs (in particular, differentially private algorithms must be randomized). In this paper, we refer to differential privacy as max-KL-stability (Definition 2.3), to emphasize the relation to the literature on algorithmic stability other notions of stability we study (KL- and TV-stability, in particular).

In this work, we extend the results of Dwork et al. along two axes. First, we give an optimal analysis of the statistical validity of max-KL stable algorithms. As a consequence, we immediately obtain the best known bounds on the sample complexity (equivalently, the convergence rate) of adaptive data analysis. Second, we generalize the connection between max-KL stability and statistical validity to a much larger family of statistics. Our proofs are also significantly simpler than those of Dwork et al., and clarify the role of different stability notions in the adaptive setting.

\footnotetext{
${ }^{1}$ See, e.g., $[\mathrm{McS}]$, although the observation itself dates back at least to 2008 (personal communication).
}

\subsection{Overview of Results}

\section{Adaptivity and Statistical Queries.}

Following the previous work on this subject $\left(\mathrm{DFH}^{+} 15 \mathrm{~b}\right)$, we formalize the problem of adaptive data analysis as follows. There is a distribution $\mathbf{P}$ over some finite universe $\mathcal{X}$, and a mechanism $\mathcal{M}$ that does not know $\mathbf{P}$, but is given a set $\mathbf{x}$ consisting of $n$ samples from $\mathbf{P}$. Using its sample, the mechanism must answer queries on $\mathbf{P}$. Here, a query $q$, coming from some family $Q$, maps a distribution $\mathbf{P}$ to a real-valued answer. The mechanism's answer $a$ to a query $q$ is $\alpha$-accurate if $|a-q(\mathbf{P})| \leq \alpha$ with high probability. Importantly, the mechanism's goal is to provide answers that "generalize" to the underlying distribution, rather than answers that are specific to its sample.

We model adaptivity by allowing a data analyst to ask a sequence of queries $q_{1}, q_{2}, \ldots, q_{k} \in Q$ to the mechanism, which responds with answers $a_{1}, a_{2}, \ldots, a_{k}$. In the adaptive setting, the query $q_{j}$ may depend on the previous queries and answers $q_{1}, a_{1}, \ldots, q_{j-1}, a_{j-1}$ arbitrarily. We say the mechanism is $\alpha$-accurate given $n$ samples for $k$ adaptively chosen queries if, with high probability, when given a vector $\mathbf{x}$ of $n$ samples from an arbitrary distribution $\mathbf{P}$, the mechanism accurately responds to any adaptive analyst that makes at most $k$ queries.

Dwork et al. $\mathrm{DFH}^{+} 15 \mathrm{~b}$ considered the family of statistical queries Kea93. A statistical query $q$ asks for the expected value of some function on random draws from the distribution. That is, the query is specified by a function $p: \mathcal{X} \rightarrow[0,1]$ and its answer is $q(\mathbf{P})=\mathbb{E}_{z \leftarrow_{\mathrm{R}} \mathbf{P}}[p(z)]$.

The most natural way to answer a statistical query is to compute the empirical answer $\mathbb{E}_{z \leftarrow_{\mathrm{R}} \mathbf{x}}[p(z)]$, which is just the average value of the function on the given sample $x \bigsqcup^{2}$ It is simple to show that when $k$ queries are specified nonadaptively (i.e. independent of previous answers), then the empirical answer is within $q(\mathbf{P}) \pm \alpha$ (henceforth, " $\alpha$-accurate") with high probability so long as the sample has size $n \gtrsim$ $\log (k) / \alpha^{2} \cdot 3^{3}$ However, when the queries can be chosen adaptively, the empirical average performs much worse. In particular, there is an algorithm (based on [DN03] that, after seeing the empirical answer to $k=O\left(\alpha^{2} n\right)$ queries drawn at random from a natural distribution, can find a query such that the empirical answer and the correct answer differ by $\alpha$. Thus, the empirical average is only guaranteed to be accurate when $n \gtrsim k / \alpha^{2}$, and so exponentially more samples are required to guarantee accuracy when the queries may be adaptive.

\section{Answering Adaptive Statistical Queries.}

Surprisingly, Dwork et al. $\mathrm{DFH}^{+} 15 \mathrm{~b}$, showed there are mechanisms that are much more effective than naïvely outputting the empirical answer. They show that "stable" mechanisms are accurate and, by applying a stable mechanism from the literature on differential privacy, they obtain a mechanisms that are accurate given only $n \gtrsim \sqrt{k} / \alpha^{2.5}$ samples, which is a significant improvement over the naïve mech-

\footnotetext{
${ }^{2}$ For convenience, we will often use $\mathbf{x}$ as shorthand for the empirical distribution over $\mathbf{x}$. We use $z \leftarrow_{\mathrm{R}} \mathbf{x}$ to mean a random element chosen from the uniform distribution over the elements of $\mathbf{x}$.

${ }^{3}$ This guarantee follows from bounding the error of each query using a Chernoff bound and then taking a union bound over all queries. The $\log k$ term corresponds to the Bonferroni correction in classical statistics.
} 
anism when $\alpha$ is not too small. (See Table 1 for more detailed statements of their results, including results that achieve an exponential improvement in the sample complexity when $|\mathcal{X}|$ is bounded.)

Our first contribution is to give a simpler and quantitatively optimal analysis of the generalization properties of stable algorithms, which immediately yields new accuracy bounds for adaptive statistical queries. In particular, we show that $n \gtrsim \sqrt{k} / \alpha^{2}$ samples suffice. Since $1 / \alpha^{2}$ samples are required to answer a single nonadaptive query, our dependence on $\alpha$ is optimal.

\section{Beyond Statistical Queries.}

Although statistical queries are surprisingly general Kea93, we would like to be able to ask more general queries on the distribution $\mathbf{P}$ that capture a wider variety of machine learning and data mining tasks. To this end, we give the first bounds on the sample complexity required to answer large numbers of adaptively chosen low-sensitivity queries and optimization queries, which we now describe.

Low-sensitivity queries are a generalization of statistical queries. A query is specified by an arbitrary function $p$ : $\mathcal{X}^{n} \rightarrow \mathbb{R}$ satisfying $\left|p(\mathbf{x})-p\left(\mathbf{x}^{\prime}\right)\right| \leq 1 / n$ for every $\mathbf{x}, \mathbf{x}^{\prime} \in \mathcal{X}^{n}$ differing on exactly one element. The query applied to the population is defined to be $q(\mathbf{P})=\mathbb{E}_{\mathbf{x} \leftarrow{ }_{\mathrm{R}} \mathbf{P}^{n}}[p(\mathbf{x})]$. Examples include distance queries (e.g. "How far is the sample from being well-clustered?") and maxima of statistical queries (e.g. "What is the classification error of the best $k$-node decision tree?")

Optimization queries are a broad generalization of lowsensitivity queries to arbitrary output domains. The query is specified by a loss function $L: \mathcal{X}^{n} \times \Theta \rightarrow \mathbb{R}$ that is lowsensitivity in its first parameter, and the goal is to output $\theta \in \Theta$ that is "best" in the sense that it minimizes the average loss. Specifically, $q(\mathbf{P})=\arg \min _{\theta \in \Theta} \mathbb{E}_{z \leftarrow{ }_{\mathrm{R}} \mathbf{P}^{n}}[L(z ; \theta)]$. An important special case is when $\Theta \subseteq \mathbb{R}^{d}$ is convex and $L$ is convex in $\theta$, which captures many fundamental regression and classification problems.

Our sample complexity bounds are summarized in Table 1

\section{Subsequent Work.}

Our bounds were applied in subsequent work of Dwork et al. $\mathrm{DFH}^{+} 15 \mathrm{c}, \mathrm{DFH}^{+} 15 \mathrm{a}$ in the analysis of their "reusable holdout" construction. Other subsequent works RZ16, WLF16 consider incomparable models of adaptive data analysis; they provide results that do not compare directly to ours.

\subsection{Overview of Techniques}

Our main result is a new proof, with optimal parameters, that a stable algorithm that provides answers to adaptive queries that are close to the empirical value on the sample gives answers that generalize to the underlying distribution. In particular, we prove:

Theorem 1.1 (Main "Transfer Theorem"). Let $\mathcal{M}$ be a mechanism that takes a sample $\mathbf{x} \in \mathcal{X}^{n}$ and answers $k$ adaptively-chosen low-sensitivity queries. Suppose that $\mathcal{M}$ satisfies the following:

\section{For every sample $\mathbf{x}, \mathcal{M}$ 's answers are $(\alpha, \alpha \beta)$-accurat $\S^{4}$}

\footnotetext{
${ }^{4}$ Accuracy is formally defined in Section 2.2. Informally, a mechanism is $(\alpha, \beta)$-accurate if every answer it produces to an adversarial adaptive sequence of queries is $\alpha$-accurate with probability at least $1-\beta$.
}

with respect to the sample $\mathbf{x}$. That is,

$$
\mathbb{P}\left[\max _{j \in k}\left|q_{j}(\mathbf{x})-a_{j}\right| \leq \alpha\right] \geq 1-\alpha \beta,
$$

where $q_{1}, \cdots, q_{k}: \mathcal{X}^{n} \rightarrow \mathbb{R}$ are the low-sensitivity queries that are asked and $a_{1}, \cdots, a_{k} \in \mathbb{R}$ are the answers given. The probability is taken only over $\mathcal{M}$ 's random coins.

2. $\mathcal{M}$ satisfies $(\alpha, \alpha \beta)$-max-KL stability (Definition 2.3 . identical to $(\alpha, \alpha \beta)$-differential privacy).

Then, if $\mathbf{x}$ consists of $n$ samples from an arbitrary distribution $\mathbf{P}$ over $\mathcal{X}, \mathcal{M}$ 's answers are $(O(\alpha), O(\beta))$-accurate with respect to $\mathbf{P}$. That is, $\mathbb{P}\left[\max _{j \in k}\left|q_{j}(\mathbf{P})-a_{j}\right| \leq O(\alpha)\right] \geq$ $1-O(\beta)$, where the probability is taken only over the choice of $\mathbf{x} \leftarrow{ }_{R} \mathbf{P}^{n}$ and $\mathcal{M}$ 's random coins.

Our actual result is somewhat more general than Theorem 1.1 We show that the population-level error of a stable algorithm is close to its error on the sample, whether or not that error is low. Put glibly: stable algorithms cannot be wrong without realizing it.

Compared to the results of $\mathrm{DFH}^{+} 15 \mathrm{~b}$, Theorem 1.1 requires a quantitatively weaker stability guarantee- $(\alpha, \alpha \beta)$ stability, instead of $\left(\alpha,(\beta / k)^{1 / \alpha}\right)$-stability. It also applies to arbitrary low-sensitivity queries as opposed to the special case of statistical queries.

Our analysis differs from that of Dwork et al. in two key ways. First, we give a better bound on the probability with which a single low-sensitivity query output by a max-KL stable algorithm has good generalization error. Second, we show a reduction from the case of many queries to the case of a single query that has no loss in parameters (in contrast, previous work took a union bound over queries, leading to a dependence on $k$, the number of queries).

Both steps rely on a thought experiment in which several "real" executions of a stable algorithm are simulated inside another algorithm, called a monitor, which outputs a function of the "real" transcripts. Because stability is closed under post-processing, the monitor is itself stable. Because it exists only as a thought experiment, the monitor can be given knowledge of the true distribution from which the data are drawn, and can use this knowledge to process the outputs of the simulated "real" runs. The monitor technique allows us to start from a basic guarantee, which states that a single query has good generalization error with constant probability, and amplify the guarantee so that $(a)$ the generalization error holds with very high probability, and (b) the guarantee holds simultaneously over all queries in a sequence. The proof of the basic guarantee follows the lines of existing proofs using algorithmic stability (e.g., DW79a]), while the monitor technique and the resulting amplification statements are new.

The amplification of success probability is the more technically sophisticated of the two key steps. The idea is to run many (about $1 / \beta$, using the notation of Theorem 1.1) copies of a stable mechanism on independently selected data sets. Each of these interactions results in a sequence of queries and answers. The monitor then selects the query and answer pair from amongst all of the sequences that has the largest error. It outputs this query as well as the index of the interaction that produced it. Our main technical lemma shows that the monitor will find a "bad" query/dataset pair (one where the 


\begin{tabular}{|c|c|c|c|}
\hline Query Type & \multicolumn{2}{|c|}{ Sample Complexity } & Time per Query \\
\cline { 2 - 4 } & $n=\tilde{O}\left(\frac{\sqrt{k}}{\alpha^{2.5}}\right)$ & $n=\tilde{O}\left(\frac{\sqrt{k}}{\alpha^{2}}\right)$ & $\operatorname{poly}(n, \log |\mathcal{X}|)$ \\
\hline Statistical $\left(k \ll n^{2}\right)$ & $n=\tilde{O}\left(\frac{\sqrt{\log |\mathcal{X}|} \cdot \log ^{3 / 2} k}{\alpha^{3.5}}\right)$ & $n=\tilde{O}\left(\frac{\sqrt{\log |\mathcal{X}|} \cdot \log k}{\alpha^{3}}\right)$ & $\operatorname{poly}(n,|\mathcal{X}|)$ \\
\hline Statistical $\left(k \gg n^{2}\right)$ & - & $n=\tilde{O}\left(\frac{\sqrt{k}}{\alpha^{2}}\right)$ & $\operatorname{poly}(n, \log |\mathcal{X}|)$ \\
\hline Low Sensitivity $\left(k \ll n^{2}\right)$ & - & $n=\tilde{O}\left(\frac{\log |\mathcal{X}| \log k}{\alpha^{3}}\right)$ & $\operatorname{poly}\left(|\mathcal{X}|^{n}\right)$ \\
\hline Low Sensitivity $\left(k \gg n^{2}\right)$ & - & $n=\tilde{O}\left(\frac{\sqrt{d k}}{\alpha^{2}}\right)$ & $\operatorname{poly}(n, d, \log |\mathcal{X}|)$ \\
\hline Convex Min. $\left(k \ll n^{2}\right)$ & - & $n=\tilde{O}\left(\frac{(\sqrt{d}+\log k) \cdot \sqrt{\log |\mathcal{X}|}}{\alpha^{3}}\right)$ & $\operatorname{poly}(n, d,|\mathcal{X}|)$ \\
\hline Convex Min. $\left(k \gg n^{2}\right)$ & & &
\end{tabular}

Table 1: Summary of Results. Here $k=$ number of queries, $n=$ number of samples, $\alpha=$ desired accuracy, $\mathcal{X}=$ universe of possible samples, $d=$ dimension of parameter space $\Theta$.

true and empirical values of the query differ) with at most constant probability. This implies that the each of the real executions outputs a bad query with probability $O(\beta)$. Relative to previous work, the resulting argument yields better bounds, applies to more general classes of queries, and even generalizes to other notions of stability.

\section{Optimality.}

In general, we cannot prove that our bounds are optimal. Even for nonadaptive statistical queries, $n \gtrsim \log (k) / \alpha^{2}$ samples are necessary, and HU14. SU15b] showed that $n \gtrsim$ $\min \{\sqrt{k}, \sqrt{\log |\mathcal{X}|}\} / \alpha$ samples are necessary to answer adaptively chosen statistical queries. However, the gap between the upper and lower bounds is still significant.

However, we can show that our connection between maxKL stability and generalization is optimal (see Section 7 for details). Moreover, for every family of queries we consider, no max-KL stable algorithm can achieve better sample complexity BUV14. BST14. Thus, any significant improvement to our bounds must come from using a weaker notion of stability or some entirely different approach.

\section{Computational Complexity.}

Throughout, we will assume that the analyst only issues queries $q$ such that the empirical answer $q(\mathbf{x})$ can be evaluated in time $\operatorname{poly}(n, \log |\mathcal{X}|)$. When $k \ll n^{2}$ our algorithms have similar running time. However, when answering $k \gg$ $n^{2}$ queries, our algorithms suffer running time at least $\operatorname{poly}(n,|\mathcal{X}|)$. Since the mechanism's input is of size $n \cdot \log |\mathcal{X}|$, these algorithms cannot be considered computationally efficient. For example, if $\mathcal{X}=\{0,1\}^{d}$ for some dimension $d$, then in the non adaptive setting $\operatorname{poly}(n, d)$ running time would suffice, whereas our algorithms require $\operatorname{poly}\left(n, 2^{d}\right)$ running time. Unfortunately, this running time is known to be optimal, as HU14, SU15b (building on hardness results in privacy [Ull13]) showed that, assuming exponentially hard one-way functions exist, any $\operatorname{poly}\left(n, 2^{o(d)}\right)$ time mechanism that answers $k=\omega\left(n^{2}\right)$ statistical queries is not even $1 / 3$-accurate.

\section{Stable / Differentially Private Mechanisms.}

Each of our results requires instantiating the mechanism with a suitable stable / differentially private algorithm. For statistical queries, the optimal mechanisms are the well known
Gaussian and Laplace Mechanisms (slightly refined by SU15a]) when $k$ is small and the Private Multiplicative Weights Mechanism HR10 when $k$ is large. For arbitrary low-sensitivity queries, the Gaussian or Laplace Mechanism is again optimal when $k$ is small, and for large $k$ we can use the Median Mechanism RR10.

When considering arbitrary search queries over an arbitrary finite range, the optimal algorithm is the Exponential Mechanism [MT07. For the special case of convex minimization queries over an infinite domain, we use the optimal algorithm of BST14 when $k$ is small, and when $k$ is large, we use an algorithm of Ull15 that accurately answers exponentially many such queries.

\section{Other Notions of Stability.}

Our techniques apply to notions of distributional stability other than max-KL/differential privacy. In particular, defining stability in terms of total variation (TV) or KL divergence $(\mathrm{KL})$ leads to bounds on the generalization error that have polynomially, rather than exponentially, decreasing tails. See Section 4 for details.

\section{PRELIMINARIES}

\subsection{Queries}

Given a distribution $\mathbf{P}$ over $\mathcal{X}$ or a sample $\mathbf{x}=\left(\mathbf{x}_{1}, \cdots, \mathbf{x}_{n}\right)$ $\in \mathcal{X}^{n}$, we would like to answer queries about $\mathbf{P}$ or $x$ from some family $Q$. We will often want to bound the "sensitivity" of the queries with respect to changing one element of the sample. To this end, we use $x \sim x^{\prime}$ to denote that $x, x^{\prime} \in \mathcal{X}^{n}$ differ on at most one entry. We will consider several different families of queries:

- Statistical Queries: These queries are specified by a function $q: \mathcal{X} \rightarrow[0,1]$, and (abusing notation) are defined as

$$
q(\mathbf{P})=\underset{z \leftarrow \mathrm{R}_{\mathrm{R}} \mathbf{P}}{\mathbb{E}}[q(z)] \quad \text { and } \quad q(\mathbf{x})=\frac{1}{n} \sum_{i \in[n]} q\left(x_{i}\right) .
$$

The error of an answer $a$ to a statistical query $q$ with respect to $\mathbf{P}$ or $\mathbf{x}$ is defined to be

$$
\operatorname{err}_{\mathbf{x}}(q, a)=a-q(\mathbf{x}) \quad \text { and } \quad \operatorname{err}^{\mathbf{P}}(q, a)=a-q(\mathbf{P}) .
$$


- $\Delta$-Sensitive Queries: For $\Delta \in[0,1], n \in \mathbb{N}$, these queries are specified by a function $q: \mathcal{X}^{n} \rightarrow \mathbb{R}$ satisfying $\left|q(x)-q\left(x^{\prime}\right)\right| \leq \Delta$ for every pair $x, x^{\prime} \in \mathcal{X}^{n}$ differing in only one entry. Abusing notation, let

$$
q(\mathbf{P})=\underset{z \leftarrow \mathbf{R} \mathbf{P}^{n}}{\mathbb{E}}[q(z)] .
$$

The error of an answer $a$ to a $\Delta$-sensitive query $q$ with respect to $\mathbf{P}$ or $\mathbf{x}$ is defined to be

$$
\begin{gathered}
\operatorname{err}_{\mathbf{x}}(q, a)=a-q(\mathbf{x}) \\
\text { and } \\
\operatorname{err}^{\mathbf{P}}(q, a)=\underset{z \leftarrow \mathbf{R}^{n}}{\mathbb{E}}\left[\operatorname{err}_{z}(q, a)\right]=a-q(\mathbf{P}) .
\end{gathered}
$$

We denote the set of all $\Delta$-sensitive queries by $Q_{\Delta}$. If $\Delta=$ $O(1 / n)$ we say the query is low sensitivity. Note that $1 / n$ sensitive queries are a strict generalization of statistical queries.

- Minimization Queries: These queries are specified by a loss function $L: \mathcal{X}^{n} \times \Theta \rightarrow \mathbb{R}$. We require that $L$ has sensitivity $\Delta$ with respect to its first parameter, that is,

$$
\sup _{\theta \in \Theta, x, x^{\prime} \in \mathcal{X}^{n}, x \sim x^{\prime}}\left|L(x ; \theta)-L\left(x^{\prime} ; \theta\right)\right| \leq \Delta .
$$

Here $\Theta$ is an arbitrary set of items (sometimes called "parameter values") among which we aim to chose the item ("parameter") with minimal loss, either with respect to a particular input data set $x$, or with respect to expectation over a distribution $\mathbf{P}$.

The error of an answer $\theta \in \Theta$ to a minimization query $L: \mathcal{X}^{n} \times \Theta \rightarrow \mathbb{R}$ with respect to $\mathbf{x}$ is defined to be

$$
\operatorname{err}_{\mathbf{x}}(L, \theta)=L(\mathbf{x}, \theta)-\min _{\theta^{*} \in \Theta} L\left(\mathbf{x}, \theta^{*}\right)
$$

and, with respect to $\mathbf{P}$, is

$$
\begin{aligned}
\operatorname{err}^{\mathbf{P}}(L, \theta) & =\underset{z \leftarrow \mathrm{R}^{n}}{\mathbb{E}}\left[\operatorname{Prr}_{z}(L, \theta)\right] \\
& =\underset{z \leftarrow{ }_{\mathrm{R}} \mathbf{P}^{n}}{\mathbb{E}}[L(z, \theta)]-\underset{z \leftarrow \leftarrow_{\mathrm{R}} \mathbf{P}^{n}}{\mathbb{E}}\left[\min _{\theta^{*} \in \Theta} L\left(z, \theta^{*}\right)\right] .
\end{aligned}
$$

Note that

$$
\min _{\theta^{*} \in \Theta} \underset{z \leftarrow \mathrm{R}^{n}}{\mathbb{E}}\left[L\left(z, \theta^{*}\right)\right] \geq \underset{z \leftarrow \mathrm{R}}{\mathbb{E}} \mathbf{P}^{n}\left[\min _{\theta^{*} \in \Theta} L\left(z, \theta^{*}\right)\right],
$$

whence

$$
\underset{z \leftarrow \mathbf{R}^{n}}{\mathbb{E}}[L(z, \theta)]-\min _{\theta^{*} \in \Theta} \underset{z \leftarrow \mathrm{R}^{n}}{\mathbb{E}}\left[L\left(z, \theta^{*}\right)\right] \leq \operatorname{err}^{\mathbf{P}}(L, \theta) .
$$

Note that minimization queries (with $\Theta=\mathbb{R}$ ) generalize low-sensitivity queries: Given a $\Delta$-sensitive $q: \mathcal{X}^{n} \rightarrow \mathbb{R}$, we can define $L(\mathbf{x} ; \theta)=|\theta-q(\mathbf{x})|$ to obtain a minimization query with the same answer.

We denote the set of minimization queries by $Q_{\text {min }}$. We highlight two special cases:

- Minimization for Finite Sets: We denote by $Q_{m i n, D}$ the set of minimization queries where $\Theta$ is finite with size at most $D$.

- Convex Minimization Queries: If $\Theta \subset \mathbb{R}^{d}$ is closed and convex and $L(\mathbf{x} ; \cdot)$ is convex on $\Theta$ for every data set $\mathbf{x}$, then the query can be answered nonprivately up to any desired error $\alpha$, in time polynomial in $d$ and $\alpha$. We denote the set of all convex minimization queries by $Q_{C M}$.

\subsection{Mechanisms for Adaptive Queries}

Our goal is to design a mechanism $\mathcal{M}$ that answers queries on $\mathbf{P}$ using only independent samples $x_{1}, \ldots, x_{n} \leftarrow_{\mathrm{R}} \mathbf{P}$. Our focus is the case where the queries are chosen adaptively according to an arbitrary rule which we model as chosen adversarially.

Specifically, $\mathcal{M}$ is a stateful algorithm that holds a collection of samples $x_{1}, \ldots, x_{n} \in \mathcal{X}$, takes a query $q$ from some family $Q$ as input, and returns an answer $a$. We require that when $x_{1}, \ldots, x_{n}$ are independent samples from $\mathbf{P}$, the answer $a$ is "close" to $q(\mathbf{P})$ in a sense that is appropriate for the family of queries. Moreover we require that this condition holds for every query in an adaptively chosen sequence $q_{1}, \ldots, q_{k}$. Formally, we define an accuracy game between a mechanism $\mathcal{M}$ and a stateful data analyst $\mathcal{A}$ in Figure 1

$\mathcal{A}$ chooses a distribution $\mathbf{P}$ over $\mathcal{X}$.

Sample $x_{1}, \ldots, x_{n} \leftarrow_{\mathrm{R}} \mathbf{P}$, let $\mathbf{x}=\left(x_{1}, \ldots, x_{n}\right)$. (Note that $\mathcal{A}$ does not know x.)

For $j=1, \ldots, k$

$\mathcal{A}$ outputs a query $q_{j} \in Q$.

$\mathcal{M}\left(\mathbf{x}, q_{j}\right)$ outputs $a_{j}$.

(As $\mathcal{A}$ and $\mathcal{M}$ are stateful, $q_{j}$ and $a_{j}$ may depend on the history $q_{1}, a_{1}, \ldots, q_{j-1}, a_{j-1}$.)

Figure 1: The Accuracy Game $\operatorname{Acc}_{n, k, Q}[\mathcal{M}, \mathcal{A}]$

Definition 2.1 (AcCuracy). A mechanism $\mathcal{M}$ is $(\alpha, \beta)$ accurate with respect to the population for $k$ adaptively chosen queries from $Q$ given $n$ samples in $\mathcal{X}$ if for every adversary $\mathcal{A}$,

$$
\underset{\operatorname{Acc}_{n, k, Q}[\mathcal{M}, \mathcal{A}]}{\mathbb{P}}\left[\max _{j \in[k]}\left|\operatorname{err}^{\mathbf{P}}\left(q_{j}, a_{j}\right)\right| \leq \alpha\right] \geq 1-\beta .
$$

We will also use a definition of accuracy relative to the sample given to the mechanism, described in Figure 2.

$\mathcal{A}$ chooses $\mathbf{x}=\left(\mathbf{x}_{1}, \ldots, \mathbf{x}_{n}\right) \in \mathcal{X}^{n}$.

For $j=1, \ldots, k$

$\mathcal{A}$ outputs a query $q_{j} \in Q$.

$\mathcal{M}\left(\mathbf{x}, q_{j}\right)$ outputs $a_{j}$.

$\left(q_{j}\right.$ and $a_{j}$ may depend on the history

$q_{1}, a_{1}, \ldots, q_{j-1}, a_{j-1}$ and on $\mathbf{x}$.)

$\begin{array}{lllll}\text { Figure 2: } & \text { The } & \text { Sample Accuracy } & \text { Game } \\ \text { SampAcc }_{n, k, Q}[\mathcal{M}, \mathcal{A}] & & & \end{array}$

Definition 2.2 (Sample Accuracy). A mechanism $\mathcal{M}$ is $(\alpha, \beta)$-accurate with respect to samples of size $n$ from $\mathcal{X}$ for $k$ adaptively chosen queries from $Q$ if for every adversary $\mathcal{A}$

$$
\underset{\operatorname{SampAcc}_{n, k, Q}[\mathcal{M}, \mathcal{A}]}{\mathbb{P}}\left[\max _{j \in[k]}\left|\operatorname{err}_{\mathbf{x}}\left(q_{j}, a_{j}\right)\right| \leq \alpha\right] \geq 1-\beta .
$$

\subsection{Max-KL Stability (a.k.a. Differential Pri- vacy)}

Informally, an algorithm is "stable" if changing one of its inputs does not change its output "too much." For our results, we will consider randomized algorithms, and require 
that changing one input does not change the distribution of the algorithm's outputs too much. With this in mind, we will define here one notion of algorithmic stability that is related to the well-known notion of KL-divergence between distributions. In Section 4.1 we will give other related notions of algorithmic stability based on different notions of closeness between distributions.

Definition 2.3 (Max-KL Stability). Let $\mathcal{W}: \mathcal{X}^{n} \rightarrow \mathcal{R}$ be a randomized algorithm. We say that $\mathcal{W}$ is $(\varepsilon, \delta)-\max -\mathrm{KL}$ stable if for every pair of samples $\mathbf{x}, \mathbf{x}^{\prime}$ that differ on exactly one element, and every $R \subseteq \mathcal{R}$,

$$
\mathbb{P}[\mathcal{W}(\mathbf{x}) \in R] \leq e^{\varepsilon} \cdot \mathbb{P}\left[\mathcal{W}\left(\mathbf{x}^{\prime}\right) \in R\right]+\delta .
$$

This notion of $(\varepsilon, \delta)$-max-KL stability is also commonly known as $(\varepsilon, \delta)$-differential privacy, however in this context we choose the term max-KL stability to emphasize the conceptual relationship between this notion and other notions of algorithmic stability that have been studied in machine learning. We also emphasise that our work has a very different motivation to the motivation of differential privacy — stable algorithms are desirable even when privacy is not a concern, such as when the data does not concern humans.

In our analysis, we will make crucial use of the fact that max-KL-stability (as well as the other notions of stability discussed in Section 4.1. is closed under post-processing.

Lemma 2.4 (Post-Processing). Let $\mathcal{W}: \mathcal{X}^{n} \rightarrow \mathcal{R}$ and $f: \mathcal{R} \rightarrow \mathcal{R}^{\prime}$ be a pair of randomized algorithms. If $\mathcal{W}$ is $(\varepsilon, \delta)$-max-KL-stable then the algorithm $f(\mathcal{W}(\mathbf{x}))$ is $(\varepsilon, \delta)$ max-KL-stable.

\subsubsection{Stability for Interactive Mechanisms}

The definition we gave above does not immediately apply to algorithms that interact with a data analyst to answer adaptively chosen queries. Such a mechanism does not simply take a sample $\mathbf{x}$ as input and produce an output. Instead, in the interactive setting, there is a mechanism $\mathcal{M}$ that holds a sample $\mathbf{x}$ and interacts with some algorithm $\mathcal{A}$. We can view this entire interaction between $\mathcal{M}$ and $\mathcal{A}$ as a single noninteractive meta algorithm that outputs the transcript of the interaction and consider the stability of that meta algorithm. Specifically, we define the algorithm $\mathcal{W}[\mathcal{M}, \mathcal{A}](x)$ that simulates the interaction between $\mathcal{M}(x)$ and $\mathcal{A}$ and outputs the messages sent between them. We say an interactive mechanism $\mathcal{M}$ is $(\varepsilon, \delta)$-max-KL stable for $k$ queries from $Q$ if for every adversary $\mathcal{A}$, the algorithm $\mathcal{W}_{n, k, Q}[\mathcal{M}, \mathcal{A}](\mathbf{x}): \mathcal{X}^{n} \rightarrow(Q \times \mathcal{R})^{k}$ is $(\varepsilon, \delta)$-max-KL stable.

\subsubsection{Composition of Max-KL Stability}

The definition above allows for adaptive composition. This follows directly from composition results of $(\varepsilon, \delta)$-differentially private algorithms. A mechanism that is $(\varepsilon, \delta)$-max-KL stable for 1 query is $(\approx \varepsilon \sqrt{k}, \approx \delta k)$-stable for $k$ adaptively chosen queries DMNS06, DRV10. More precisely, for every $0 \leq \varepsilon \leq 1$ and $\delta, \delta^{\prime}>0$, if a mechanism that is $(\varepsilon, \delta)$-maxKL stable for 1 query is used to answer $k$ adaptively chosen queries, it remains $\left(\varepsilon \sqrt{k \log \left(1 / \delta^{\prime}\right)}+2 \varepsilon^{2} k, \delta^{\prime}+k \delta\right)$-max-KL stable DRV10.

\section{MAX-KL STABILITY TO ACCURACY FOR LOW-SENSITIVITY QUERIES}

In this section we prove our main result that any mechanism that is both accurate with respect to the sample and satisfies max-KL stability (with suitable parameters) is also accurate with respect to the population. The proof proceeds in two mains steps. First, we prove a lemma that says that there is no max-KL stable mechanism that takes several independent sets of samples from the distribution and finds a query and a set of samples such that the answer to that query on that set of samples is very different from the answer to that query on the population. In Section 3.1 we prove this lemma for the simpler case of statistical queries and then in 3.2 we extend the proof to the more general case of low-sensitivity queries.

The second step is to introduce a monitoring algorithm. This monitoring algorithm will simulate the interaction between the mechanism and the adversary on multiple independent sets of samples. It will then output the least accurate query across all the different interactions. We show that if the mechanism is stable then the monitoring algorithm is also stable. By choosing the number of sets of samples appropriately, we ensure that if the mechanism has even a small probability of being inaccurate in a given interaction, then the monitor will have a constant probability of finding an inaccurate query in one of the interactions. By the lemma proven in the first step, no such monitoring algorithm can satisfy max-KL stability, therefore every stable mechanism must be accurate with high probability.

\subsection{Warmup: A Multi-Sample De-Correlated Expectation Lemma for SQs}

As a warmup, in this section we give a simpler version of our main lemma for the case of statistical queries and multiple samples. The multi-sample de-correlated expectation lemma says that a max-KL stable algorithm cannot take a collection of samples $\mathbf{x}_{1}, \ldots, \mathbf{x}_{T}$ and output a pair $(q, t)$ such that $q(\mathbf{P})$ and $q\left(\mathbf{x}_{t}\right)$ differ significantly in expectation.

Lemma 3.1. Let $\mathcal{W}:\left(\mathcal{X}^{n}\right)^{T} \rightarrow Q \times[T]$ be $(\varepsilon, \delta)-\max -K L$ stable where $Q$ is the class statistical queries $q: \mathcal{X} \rightarrow[0,1]$. Let $\mathbf{P}$ be a distribution on $\mathcal{X}$ and let $\mathbf{X}=\left(\mathbf{x}_{1}, \ldots, \mathbf{x}_{T}\right) \leftarrow_{R}$ $\left(\mathbf{P}^{n}\right)^{T}$. Then

$\left|\underset{\mathbf{X}, \mathcal{W}}{\mathbb{E}}[q(\mathbf{P}) \mid(q, t)=\mathcal{W}(\mathbf{X})]-\underset{\mathbf{X}, \mathcal{W}}{\mathbb{E}}\left[q\left(\mathbf{x}_{t}\right) \mid(q, t)=\mathcal{W}(\mathbf{X})\right]\right| \leq$ $e^{\varepsilon}-1+T \delta$.

Proof of Lemma 3.1. Before giving the proof, we set up some notation. Let $\mathbf{X}=\left(\mathbf{x}_{1}, \ldots, \mathbf{x}_{T}\right)$ be a set of $T$ samples where each sample $\mathbf{x}_{t}=\left(x_{t, 1}, \ldots, x_{t, n}\right)$. For a single element $x^{\prime} \in \mathcal{X}$, and a pair of indices $(m, i) \in[T] \times[n]$, we use $\mathbf{X}_{(m, i) \rightarrow x^{\prime}}$ to denote the new set of $T$ samples where the $i$-th element of the $m$-th sample of $\mathbf{X}$ has been replaced by the element $x^{\prime}$. We can now calculate

$$
\begin{aligned}
& \underset{\mathbf{X}, \mathcal{W}}{\mathbb{E}}\left[q\left(\mathbf{x}_{t}\right) \mid(q, t)=\mathcal{W}(\mathbf{X})\right] \\
= & \frac{1}{n} \sum_{i=1}^{n} \sum_{m=1}^{T} \underset{\mathbf{X}, \mathcal{W}}{\mathbb{E}}\left[\mathbf{1}_{\{t=m\}} \cdot q\left(x_{m, i}\right) \mid(q, t)=\mathcal{W}(\mathbf{X})\right] \\
= & \frac{1}{n} \sum_{i=1}^{n} \sum_{m=1}^{T} \int_{0}^{1} \underset{\mathbf{X}, \mathcal{W}}{\mathbb{P}}\left[\mathbf{1}_{\{t=m\}} q\left(x_{m, i}\right) \geq z \mid(q, t)=\mathcal{W}(\mathbf{X})\right] \mathrm{d} z
\end{aligned}
$$


Now we can apply $(\varepsilon, \delta)$-max-KL stability.

$$
\begin{aligned}
\leq & \frac{1}{n} \sum_{i=1}^{n} \sum_{m=1}^{T} \int_{0}^{1}\left(e^{\varepsilon} .\right. \\
& \left.\underset{\mathbf{X}, \mathcal{W}}{\mathbb{P}}\left[\mathbf{1}_{\{t=m\}} q\left(x_{m, i}\right) \geq z \mid \quad(q, t)=\mathcal{W}\left(\mathbf{X}_{(m, i) \rightarrow x^{\prime}}\right)\right]+\delta\right) \mathrm{d} z \\
= & \frac{1}{n} \sum_{i=1}^{n} \sum_{m=1}^{T}\left(e^{\varepsilon} .\right. \\
& \left.\underset{x^{\prime}, \mathbf{X}, \mathcal{W}}{\mathbb{E}}\left[\mathbf{1}_{\{t=m\}} \cdot q\left(x_{m, i}\right) \mid(q, t)=\mathcal{W}\left(\mathbf{X}_{(m, i) \rightarrow x^{\prime}}\right)\right]+\delta\right) \\
= & \frac{1}{n} \sum_{i=1}^{n} \sum_{m=1}^{T}\left(e^{\varepsilon} \underset{x^{\prime}, \mathbf{X}, \mathcal{W}}{\mathbb{E}}\left[\mathbf{1}_{\{t=m\}} \cdot q\left(x^{\prime}\right) \mid(q, t)=\mathcal{W}(\mathbf{X})\right]+\delta\right)
\end{aligned}
$$

(the pairs $\left(x_{m, i}, \mathbf{X}_{(m, i) \rightarrow x^{\prime}}\right)$ and $\left(x^{\prime}, \mathbf{X}\right)$ are identially distributed)

$$
\begin{aligned}
& =e^{\varepsilon} \cdot \underset{x^{\prime}, \mathbf{X}, \mathcal{W}}{\mathbb{E}}\left[q\left(x^{\prime}\right) \mid(q, t)=\mathcal{W}(\mathbf{X})\right]+T \delta \\
& =e^{\varepsilon} \cdot \underset{\mathbf{X}, \mathcal{W}}{\mathbb{E}}[q(\mathbf{P}) \mid(q, t)=\mathcal{W}(\mathbf{X})]+T \delta \\
& \leq \underset{\mathbf{X}, \mathcal{W}}{\mathbb{E}}[q(\mathbf{P}) \mid(q, t)=\mathcal{W}(\mathbf{X})]+e^{\varepsilon}-1+T \delta
\end{aligned}
$$$$
\text { (since } q(\mathbf{P}) \in[0,1] \text { ) }
$$

An identical argument shows that

$$
\begin{aligned}
& \underset{\mathbf{X}, \mathcal{W}}{\mathbb{E}}\left[q\left(\mathbf{x}_{t}\right) \mid(q, t)=\mathcal{W}(\mathbf{X})\right] \\
& \geq \underset{\mathbf{X}, \mathcal{W}}{\mathbb{E}}[q(\mathbf{P}) \mid(q, t)=\mathcal{W}(\mathbf{X})]+\left(e^{-\varepsilon}-1\right)-T \delta
\end{aligned}
$$

\subsection{A Multi-Sample De-Correlated Expecta- tion Lemma}

Here, we give the most general de-correlated expectation lemma that considers multiple samples and applies to the more general class of low-sensitivity queries.

Lemma 3.2 (Main TeChnical Lemma). Let $\mathcal{W}:\left(\mathcal{X}^{n}\right)^{T} \rightarrow$ $Q_{\Delta} \times[T]$ be $(\varepsilon, \delta)$-max-KL stable where $Q_{\Delta}$ is the class of $\Delta$-sensitive queries $q: \mathcal{X}^{n} \rightarrow \mathbb{R}$. Let $\mathbf{P}$ be a distribution on $\mathcal{X}$ and let $\mathbf{X}=\left(\mathbf{x}_{1}, \ldots, \mathbf{x}_{T}\right) \leftarrow_{R}\left(\mathbf{P}^{n}\right)^{T}$. Then

$\left|\underset{\mathbf{X}, \mathcal{W}}{\mathbb{E}}[q(\mathbf{P}) \mid(q, t)=\mathcal{W}(\mathbf{X})]-\underset{\mathbf{X}, \mathcal{W}}{\mathbb{E}}\left[q\left(\mathbf{x}_{t}\right) \mid(q, t)=\mathcal{W}(\mathbf{X})\right]\right| \leq$ $2\left(e^{\varepsilon}-1+T \delta\right) \Delta n$.

We remark that if we use the weaker assumption that $\mathcal{W}$ is $\left(e^{\varepsilon}-1+\delta\right)$-TV stable, (defined in Section 4.1), then we would obtain the same conclusion but with the weaker bound of $2 T\left(e^{\varepsilon}-1+\delta\right) \Delta n$. The advantage of using the stronger definition of max-KL stability is that we only have to decrease $\delta$ with $T$ and not $\varepsilon$. This advantage is crucial because algorithms satisfying $(\varepsilon, \delta)$-max-KL stability necessarily have a linear dependence on $1 / \varepsilon$ but only a polylogarithmic dependence on $1 / \delta$.

The proof of Lemma 3.2 is similar to that of Lemma 3.1 However, instead of directly splitting up a sum and using the fact that the summands are bounded, we must expand the expectation as a telescoping sum and argue that each difference is bounded due to the low sensitivity constraint.

\subsection{From Multi-Sample De-Correlated Expec- tation to Accuracy}

Now that we have Lemma 3.2 , we can prove the following result that max-KL stable mechanisms that are accurate with respect to their sample are also accurate with respect to the population from which that sample was drawn.

Theorem 3.3 (Main Transfer Theorem). Let $Q$ be $a$ family of $\Delta$-sensitive queries on $\mathcal{X}$. Assume that, for some $\alpha, \beta \in(0, .1), \mathcal{M}$ is

1. $(\varepsilon=\alpha / 64 \Delta n, \delta=\alpha \beta / 32 \Delta n)$-max-KL stable for $k$ adaptively chosen queries from $Q$ and

2. $\left(\alpha^{\prime}=\alpha / 8, \beta^{\prime}=\alpha \beta / 16 \Delta n\right)$-accurate with respect to its sample for $n$ samples from $\mathcal{X}$ for $k$ adaptively chosen queries from $Q$.

Then $\mathcal{M}$ is $(\alpha, \beta)$-accurate with respect to the population for $k$ adaptively chosen queries from $Q$ given $n$ samples from $\mathcal{X}$.

The key step in the proof is to define a monitoring algorithm that takes $T$ separate samples $\mathbf{X}=\left(\mathbf{x}_{1}, \ldots, \mathbf{x}_{T}\right)$ and for each sample $\mathbf{x}_{t}$, simulates an independent interaction between $\mathcal{M}\left(\mathbf{x}_{t}\right)$ and $\mathcal{A}$. This monitoring algorithm then outputs the query with the largest error across all of the queries and interactions ( $k T$ queries in total). Since changing one input to $\mathbf{X}$ only affects one of the simulations, the monitoring algorithm will be stable so long as $\mathcal{M}$ is stable, without any loss in the stability parameter. On the other hand, if $\mathcal{M}$ has even a small chance $\beta$ of answering a query with large error, then if we simulate $T \approx 1 / \beta$ independent interactions, there is a constant probability that at least one of the simulations results in a query with large error. Thus, the monitor will be a stable algorithm that outputs a query with large error in expectation. By the multi-sample de-correlated expectation lemma, such a monitor is impossible, which implies that $\mathcal{M}$ has probability $\leq \beta$ of answering any query with large error.

Proof of Theorem 3.3 . Let $\mathcal{M}$ be an interactive mechanism. Let $\mathcal{A}$ be an analyst and let $\mathbf{P}$ be the distribution chosen by $\mathcal{A}$. We define the following monitoring algorithm.

$$
\mathcal{W}(\mathbf{X})=\mathcal{W}_{\mathbf{P}}[\mathcal{M}, \mathcal{A}](\mathbf{X}):
$$

Input: $\mathbf{X}=\left(\mathbf{x}_{1}, \ldots, \mathbf{x}_{T}\right) \in\left(\mathcal{X}^{n}\right)^{T}$

For $t=1, \ldots, T$ :

Simulate $\mathcal{M}\left(\mathbf{x}_{t}\right)$ and $\mathcal{A}$ interacting, let $q_{t, 1}, \ldots, q_{t, k} \in Q$ be the queries of $\mathcal{A}$ and let $a_{t, 1}, \ldots, a_{t, k} \in \mathbb{R}$ be the corresponding Let answers of $\mathcal{M}$.

$$
\left(j^{*}, t^{*}\right)=\underset{j \in[k], t \in[T]}{\arg \max }\left|\operatorname{err}^{\mathbf{P}}\left(q_{t, j}, a_{t, j}\right)\right| .
$$

If $a_{t^{*}, j^{*}}-q_{t^{*}, j^{*}}(\mathbf{P}) \geq 0$, let $q^{*}=q_{t^{*}, j^{*}}$, otherwise let $q^{*}=-q_{t^{*}, j^{*}}$. ( $Q_{\Delta}$ is closed under negation.)

Output: $\left(q^{*}, t^{*}\right)$.

If $\mathcal{M}$ is stable then so is $\mathcal{W}$, and this fact follows easily from the post-processing lemma (Lemma 2.4): 
Claim 3.4. For every $\varepsilon, \delta \geq 0$, if the mechanism $\mathcal{M}$ is $(\varepsilon, \delta)$-max-KL stable for $k$ adaptively chosen queries from $Q$, then for every $\mathbf{P}$ and $\mathcal{A}$, the monitor $\mathcal{W}_{\mathbf{P}, k, Q}[\mathcal{M}, \mathcal{A}]$ is $(\varepsilon, \delta)$-max-KL stable.

Proof. If $\mathcal{M}$ is $(\varepsilon, \delta)$-max-KL stable for $k$ adaptively chosen queries from $Q$ then for every analyst $\mathcal{A}$ who asks $k$ queries from $Q$, and every $t$ the algorithm $\mathcal{W}^{\prime}\left(\mathbf{x}_{t}\right)$ that simulates the interaction between $\mathcal{M}\left(\mathbf{x}_{t}\right)$ and $\mathcal{A}$ and outputs the resulting query-answer pairs is $(\varepsilon, \delta)$-max-KL stable. From this, it follows that the algorithm $\mathcal{W}^{\prime}(\mathbf{X})$ that simulates the interactions between $\mathcal{M}\left(\mathbf{x}_{t}\right)$ and $\mathcal{A}$ for every $t=1, \ldots, T$ and outputs the resulting query-answer pairs is $(\varepsilon, \delta)$-maxKL stable. To see this, observe that if $\mathbf{X}, \mathbf{X}^{\prime}$ differ only on one subsample $\mathbf{x}_{t}$, then for every $t^{\prime} \neq t, \mathbf{x}_{t^{\prime}}=\mathbf{x}_{t^{\prime}}^{\prime}$ and thus the query-answer pairs corresponding to subsample $t^{\prime}$ are identically distributed regardless of whether we use $\mathbf{X}$ or $\mathbf{X}^{\prime}$ as input to $\mathcal{W}$.

Observe that the algorithm $\mathcal{W}$ defined above is simply a post-processing of these $k T$ query-answer pairs. That is, $\left(q^{*}, t^{*}\right)$ depends only on $\left\{\left(q_{t, j}, a_{t, j}\right\}_{t \in[T], j \in[k]}\right.$ and $\mathbf{P}$, and not on $\mathbf{X}$. Thus, by Lemma $2.4 \mathcal{W}$ is $(\varepsilon, \delta)$-max-KL stable.

We will use the $\mathcal{W}$ with $T=\lfloor 1 / \beta\rfloor$. In light of Claim 3.4 and our assumption that $\mathcal{M}$ is $(\varepsilon, \delta)$-max-KL stable, we can apply Lemma 3.2 to obtain

$$
\begin{aligned}
& \left|\underset{\mathbf{X}, \mathcal{W}}{\mathbb{E}}\left[q^{*}(\mathbf{P})-q^{*}\left(\mathbf{x}_{t^{*}}\right) \mid\left(q^{*}, t^{*}\right)=\mathcal{W}(\mathbf{X})\right]\right| \\
& \quad \leq 2\left(e^{\alpha / 64 \Delta n}-1+T\left(\frac{\alpha \beta}{32 \Delta n}\right)\right) \Delta n \leq \alpha / 8 .
\end{aligned}
$$

To complete the proof, we show that if $\mathcal{M}$ is not $(\alpha, \beta)$ accurate with respect to the population $\mathbf{P}$, then (1) cannot hold. To do so, we need the following natural claim about the output of the monitor.

Claim 3.5. $\underset{\mathbf{X}, \mathcal{W}}{\mathbb{P}}\left[q^{*}(\mathbf{P})-a_{q^{*}}>\alpha\right]>1-(1-\beta)^{T}$, and $q^{*}(\mathbf{P})-a_{q^{*}} \geq 0$, where $a_{q^{*}}$ is the answer to $q^{*}$ produced during the simulation. $[T]$,

Proof. Since $\mathcal{M}$ fails to be $(\alpha, \beta)$-accurate, for every $t \in$

$$
\underset{\mathbf{x}_{t}, \mathcal{M}}{\mathbb{P}}\left[\max _{j \in[k]}\left|q_{t, j}(\mathbf{P})-a_{t, j}\right|>\alpha\right]>\beta .
$$

We obtain the claim from (2) by using the fact that the $T$ sets of query-answer pairs corresponding to different subsamples $\mathbf{x}_{1}, \ldots, \mathbf{x}_{T}$ are independent. That is, the random variables $\max _{j \in[k]}\left|q_{t, j}(\mathbf{P})-a_{t, j}\right|$ indexed by $t \in[T]$ are independent. Since $q^{*}(\mathbf{P})-a_{q^{*}}$ is simply the maximum of these independent random variables, the first part of the claim follows. Also, by construction, $\mathcal{W}$ ensures that

$$
q^{*}(\mathbf{P})-a_{q^{*}} \geq 0
$$

Claim 3.6. If $\mathcal{M}$ is $\left(\alpha^{\prime}, \beta^{\prime}\right)$-accurate for the sample but not $(\alpha, \beta)$-accurate for the population, then

$$
\left|\underset{\mathbf{X}, \mathcal{W}}{\mathbb{E}}\left[q^{*}(\mathbf{P})-q^{*}\left(\mathbf{x}_{t^{*}}\right) \mid\left(q^{*}, t^{*}\right)=\mathcal{W}(\mathbf{X})\right]\right| \geq \alpha / 4
$$

Proof. Now we can calculate

$$
\begin{aligned}
& \left|\underset{\mathbf{X}, \mathcal{W}}{\mathbb{E}}\left[q^{*}(\mathbf{P})-q^{*}\left(\mathbf{x}_{t^{*}}\right) \mid\left(q^{*}, t^{*}\right)=\mathcal{W}(\mathbf{X})\right]\right| \\
= & \mid \underset{\mathbf{X}, \mathcal{W}}{\mathbb{E}}\left[q^{*}(\mathbf{P})-a_{q^{*}} \mid\left(q^{*}, t^{*}\right)=\mathcal{W}(\mathbf{X})\right] \\
& +\underset{\mathbf{X}, \mathcal{W}}{\mathbb{E}}\left[a_{q^{*}}-q^{*}\left(\mathbf{x}_{t^{*}}\right) \mid\left(q^{*}, t^{*}\right)=\mathcal{W}(\mathbf{X})\right] \mid \\
\geq & \left|\underset{\mathbf{X}, \mathcal{W}}{\mathbb{E}}\left[q^{*}(\mathbf{P})-a_{q^{*}} \mid\left(q^{*}, t^{*}\right)=\mathcal{W}(\mathbf{x})\right]\right| \\
& -\left|\underset{\mathbf{X}, \mathcal{W}}{\mathbb{E}}\left[a_{q^{*}}-q^{*}\left(\mathbf{x}_{t^{*}}\right) \mid q^{*}=\mathcal{W}(\mathbf{x})\right]\right| \\
\geq & \alpha\left(1-(1-\beta)^{T}\right)-\left|\underset{\mathbf{X}, \mathcal{W}}{\mathbb{E}}\left[a_{q^{*}}-q^{*}\left(\mathbf{x}_{t^{*}}\right) \mid\left(q^{*}, t^{*}\right)=\mathcal{W}(\mathbf{X})\right]\right| \\
\geq & \alpha\left(1-(1-\beta)^{T}\right)-\left(\alpha / 8+2 T\left(\frac{\alpha \beta}{16 \Delta n}\right) \Delta n\right) \\
\geq & \alpha / 2-(\alpha / 8+\alpha / 8)=\alpha / 4 \quad(T=\lfloor 1 / \beta\rfloor .)
\end{aligned}
$$

Line (4) follows from two observations. First, since $\mathcal{M}$ is assumed to be $(\alpha / 8, \alpha \beta / 16 \Delta n)$-accurate for one sample, by a union bound, it is simultaneously $(\alpha / 8, T(\alpha \beta / 16 \Delta n))$ accurate for all of the $T$ samples. Thus, we have $a_{q^{*}}-$ $q^{*}\left(\mathbf{x}_{t^{*}}\right) \leq \alpha^{\prime}$ except with probability at most $T(\alpha \beta / 16 \Delta n)$. Second, since $q^{*}$ is a $\Delta$-sensitive query, we always have $a_{q^{*}}-q^{*}\left(\mathbf{x}_{t^{*}}\right) \leq 2 \Delta n 5^{5}$

Thus, if $\mathcal{M}$ is not $(\alpha, \beta)$-accurate for the population, we will obtain a contradiction to (1). This completes the proof.

\section{OTHER NOTIONS OF STABILITY AND ACCURACY ON AVERAGE}

Definition 4.2 gives one notion of stability, namely maxKL stability. However, this is by no means the only way to formalise stability for our purposes. In this section we consider other notions of stability and the advantages they have.

\subsection{Other Notions of Algorithmic Stability}

We will define here other notions of algorithmic stability, and in Section 4.2. we will show that such notions can provide expected guarantees for generalization error which can be used to achieve accuracy on average.

Definition 4.1 (TV-Stability). Let $\mathcal{W}: \mathcal{X}^{n} \rightarrow \mathcal{R}$ be a randomized algorithm. We say that $\mathcal{W}$ is $\varepsilon-\mathrm{TV}$ stable if for every pair of samples that differ on exactly one element,

$$
\begin{aligned}
& \mathrm{d}_{\mathrm{TV}}\left(\mathcal{W}(\mathbf{x}), \mathcal{W}\left(\mathbf{x}^{\prime}\right)\right) \\
& =\sup _{R \subseteq \mathcal{R}}\left|\mathbb{P}[\mathcal{W}(\mathbf{x}) \in R]-\mathbb{P}\left[\mathcal{W}\left(\mathbf{x}^{\prime}\right) \in R\right]\right| \leq \varepsilon .
\end{aligned}
$$

Definition 4.2 (KL-Stability). Let $\mathcal{W}: \mathcal{X}^{n} \rightarrow \mathcal{R}$ be a randomized algorithm. We say that $\mathcal{W}$ is $\varepsilon$-KL-stable if for every pair of samples $\mathbf{x}, \mathbf{x}^{\prime}$ that differ on exactly one element,

$$
\underset{r \leftarrow R_{R} \mathcal{W}(\mathbf{x})}{\mathbb{E}}\left[\log \left(\frac{\mathbb{P}[\mathcal{W}(\mathbf{x})=r]}{\mathbb{P}\left[\mathcal{W}\left(\mathbf{x}^{\prime}\right)=r\right]}\right)\right] \leq 2 \varepsilon^{2}
$$

\footnotetext{
${ }^{5}$ Without loss of generality, the answers of $\mathcal{M}$ can be truncated to an interval of width $2 \Delta n$ that contains the correct answer $q^{*}\left(\mathbf{x}_{t^{*}}\right)$. Doing so will ensure $\left|a_{q^{*}}-q^{*}\left(\mathbf{x}_{t^{*}}\right)\right| \leq 2 \Delta n$.
} 
The post-processing property of Max-KL stability (Lemma 2.4 in Section 2.3 also applies to the two stability notions above.

Lemma 4.3 (Stability Notions Preserved under PostProcessing). Let $\mathcal{W}: \mathcal{X}^{n} \rightarrow \mathcal{R}$ and $f: \mathcal{R} \rightarrow \mathcal{R}^{\prime}$ be a pair of randomized algorithms. If $\mathcal{W}$ is $\{\varepsilon-T V, \varepsilon-K L,(\varepsilon, \delta)$ max-KL\}-stable then the algorithm $f(\mathcal{W}(\mathbf{x}))$ is $\{\varepsilon-T V$, $\varepsilon$ $K L,(\varepsilon, \delta)$-max-KL\}-stable.

\section{Relationships Between Stability Notions.}

$\varepsilon$-KL stability implies $\varepsilon$-TV stability by Pinsker's inequality. The relationship between max-KL stability defined in Section 2.3 and the above notions is more subtle. When $\varepsilon \leq 1,(\varepsilon, 0)$-max-KL stability implies $\varepsilon$-KL stability and thus also $\varepsilon$-TV stability. When $\varepsilon \leq 1$ and $\delta>0,(\varepsilon, \delta)$-maxKL stability implies $(2 \varepsilon+\delta)$-TV stability. It also implies that $\mathcal{M}$ is "close" to satisfying $2 \varepsilon-\mathrm{KL}$ stability (cf. DRV10. for more discussion of these notions).

As in Section 2.3.1, we define TV-stability and KL-stability of an interactive mechanism $\mathcal{M}$ via a noninteractive mechanism that simulates the interaction between $\mathcal{M}$ and an adversary $\mathcal{A}$. The definition for these notions of stability is precisely analogous to the interactive definition for max-KL stability.

As with max-KL stability, both notions above allow for adaptive composition. In fact, $\varepsilon$-TV stability composes linearly - a mechanism that is $\varepsilon$-TV stable for 1 query is $\varepsilon k$ stable for $k$ queries. The advantage of the stronger notions of $\mathrm{KL}$ and max-KL stability is that they have a stronger composition. A mechanism that is $\varepsilon$-KL stable for 1 query is $(\varepsilon \sqrt{k})$-stable for $k$ queries.

\subsection{From TV Stability to Accuracy on Aver- age}

In this section we show that TV stable algorithms guarantee a weaker notion of accuracy on average for adaptively chosen queries.

\subsection{Accuracy on Average}

In Section 2.2 we defined accurate mechanisms to be those that answer accurately (either with respect to the population or the sample) with probability close to 1 . In this section we define a relaxed notion of accuracy that only requires low error in expectation over the coins of $\mathcal{M}$ and $\mathcal{A}$.

Definition 4.4 (Average Accuracy). A mechanism $\mathcal{M}$ is $\alpha$-accurate on average with respect to the population for $k$ adaptively chosen queries from $Q$ given $n$ samples in $\mathcal{X}$ if for every adversary $\mathcal{A}$,

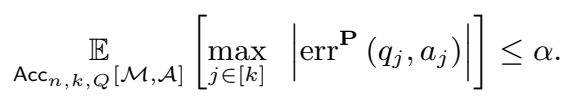

We will also use a definition of accuracy relative to the sample given to the mechanism:

Definition 4.5 (SAmple AcCuracy on Average). A mechanism $\mathcal{M}$ is $\alpha$-accurate on average with respect to samples of size $n$ from $\mathcal{X}$ for $k$ adaptively chosen queries from $Q$ if for every adversary $\mathcal{A}$,

$$
\underset{\operatorname{SampAcc}_{n, k, Q}[\mathcal{M}, \mathcal{A}]}{\mathbb{E}}\left[\max _{j \in[k]}\left|\operatorname{err}_{\mathbf{x}}\left(q_{j}, a_{j}\right)\right|\right] \leq \alpha .
$$

\subsubsection{A De-Correlated Expectation Lemma}

Towards our goal of proving that TV stability implies accuracy on average in the adaptive setting, we first prove a lemma saying that TV stable algorithms cannot output a low-sensitivity query such that the sample has large error for that query. In the next section we will show how this lemma implies accuracy on average in the adaptive setting.

Lemma 4.6. Let $\mathcal{W}: \mathcal{X}^{n} \rightarrow Q_{\Delta}$ be an $\varepsilon-T V$ stable randomized algorithm. Recall $Q_{\Delta}$ is the family of $\Delta$-sensitive queries $q: \mathcal{X}^{n} \rightarrow \mathbb{R}$. Let $\mathbf{P}$ be a distribution on $\mathcal{X}$ and let $\mathbf{x} \leftarrow{ }_{R} \mathbf{P}^{n}$. Then

$$
|\underset{\mathbf{x}, \mathcal{W}}{\mathbb{E}}[q(\mathbf{P}) \mid q=\mathcal{W}(\mathbf{x})]-\underset{\mathbf{x}, \mathcal{W}}{\mathbb{E}}[q(\mathbf{x}) \mid q=\mathcal{W}(\mathbf{x})]| \leq 2 \varepsilon \Delta n .
$$

Proof. The proof proceeds via a sequence of intermediate samples. Let $\mathbf{x}^{\prime} \leftarrow_{\mathrm{R}} \mathbf{P}^{n}$ be independent of $\mathbf{x}$. For $\ell \in\{0,1, \ldots, n\}$, we define $\mathbf{x}^{\ell}=\left(x_{1}^{\ell}, \ldots, x_{n}^{\ell}\right) \in \mathcal{X}^{n}$ by

$$
x_{i}^{\ell}= \begin{cases}x_{i} & i>\ell \\ x_{i}^{\prime} & i \leq \ell\end{cases}
$$

By construction, $\mathbf{x}^{0}=\mathbf{x}$ and $\mathbf{x}^{n}=\mathrm{x}^{\prime}$, and intermediate samples $\mathbf{x}^{\ell}$ interpolate between $\mathbf{x}$ and $\mathbf{x}^{\prime}$. Moreover, $\mathbf{x}^{\ell}$ and $\mathbf{x}^{\ell+1}$ differ in at most one entry, so that we can use the stability condition to relate $\mathcal{W}\left(\mathbf{x}^{\ell}\right)$ and $\mathcal{W}\left(\mathbf{x}^{\ell+1}\right)$.

For every $\ell \in[n]$, we define $B^{\ell}: \mathcal{X}^{n} \times \mathcal{X}^{n} \rightarrow \mathbb{R}$ by

$$
B^{\ell}(\mathbf{x}, \mathbf{z})=q(\mathbf{z})-q\left(\mathbf{z}_{-\ell}\right)+\Delta, \text { where } q=\mathcal{W}(\mathbf{x}) .
$$

Here, $\mathbf{z}_{-\ell}$ is $\mathbf{z}$ with the $\ell$-th element replaced by some arbitrary fixed element of $\mathcal{X}$.

Now we can write

$$
\begin{aligned}
& |\underset{\mathbf{x}, \mathcal{W}}{\mathbb{E}}[q(\mathbf{P})-q(\mathbf{x}) \mid q=\mathcal{W}(\mathbf{x})]| \\
= & \left|\underset{\mathbf{x}, \mathbf{x}^{\prime}, \mathcal{W}}{\mathbb{E}}\left[q\left(\mathbf{x}^{\prime}\right)-q(\mathbf{x}) \mid q=\mathcal{W}(\mathbf{x})\right]\right| \\
= & \left|\sum_{\ell=1}^{n} \underset{\mathbf{x}, \mathbf{x}^{\prime}, \mathcal{W}}{\mathbb{E}}\left[q\left(\mathbf{x}^{\ell}\right)-q\left(\mathbf{x}^{\ell-1}\right) \mid q=\mathcal{W}(\mathbf{x})\right]\right| \\
\leq & \sum_{\ell=1}^{n}\left|\underset{\mathbf{x}, \mathbf{x}^{\prime}, \mathcal{W}}{\mathbb{E}}\left[q\left(\mathbf{x}^{\ell}\right)-q\left(\mathbf{x}^{\ell-1}\right) \mid q=\mathcal{W}(\mathbf{x})\right]\right| \\
= & \sum_{\ell \in[n]} \mid \underset{\mathbf{x}, \mathbf{x}^{\prime}, \mathcal{W}}{\mathbb{E}}\left[\left(q\left(\mathbf{x}^{\ell}\right)-q\left(\mathbf{x}_{-\ell}^{\ell}\right)+\Delta\right)\right. \\
& \left.-\left(q\left(\mathbf{x}^{\ell-1}\right)-q\left(\mathbf{x}_{-\ell}^{\ell-1}\right)+\Delta\right) \mid q=\mathcal{W}(\mathbf{x})\right] \mid \\
= & \sum_{\ell \in[n]}\left|\underset{\mathbf{x}, \mathbf{x}^{\prime}, \mathcal{W}}{\mathbb{E}}\left[B^{\ell}\left(\mathbf{x}^{\ell}, \mathbf{x}^{\ell}\right)-B^{\ell}\left(\mathbf{x}, \mathbf{x}^{\ell-1}\right)\right]\right| .
\end{aligned}
$$

(Definition of $B$ )

Thus, to prove the lemma, it suffices to show that for every $\ell \in[n]$,

$$
\left|\underset{\mathbf{x}, \mathbf{x}^{\prime}, \mathcal{W}}{\mathbb{E}}\left[B^{\ell}\left(\mathbf{x}, \mathbf{x}^{\ell}\right)-B^{\ell}\left(\mathbf{x}, \mathbf{x}^{\ell-1}\right)\right]\right| \leq 2 \Delta \varepsilon
$$

To complete the proof, we will need a few observations. First, since $q$ is $\Delta$-sensitive, for every $\ell, \mathbf{x}, \mathbf{z}$, we have $0 \leq$ $B^{\ell}(\mathbf{x}, \mathbf{z}) \leq 2 \Delta$. 
Second, observe that since $\mathcal{W}$ is assumed to be $\varepsilon$-TV stable, by the post-processing lemma (Lemma 2.4 $B^{\ell}(\mathbf{x}, \mathbf{z})$ is $\varepsilon$-TV stable with respect to its first parameter $\mathbf{x}$.

Finally, observe that the random variables $\mathbf{x}^{0}, \ldots, \mathbf{x}^{n}$ are identically distributed (although not independent). That is, every $\mathbf{x}^{\ell}$ consists of $n$ independent draws from $\mathbf{P}$. Moreover, for every $\ell$, the pairs $\left(\mathbf{x}, \mathbf{x}^{\ell}\right)$ and $\left(\mathbf{x}^{\ell}, \mathbf{x}\right)$ are identically distributed. Specifically, the first component is $n$ independent samples from $\mathbf{P}$ and the second component is equal to the first component with a subset of the entries replaced by new independent samples from $\mathbf{P}$.

Combining, the second and third observation with the triangle inequality, we have

$$
\begin{aligned}
& \mathrm{d}_{\mathrm{TV}}\left(B^{\ell}\left(\mathbf{x}, \mathbf{x}^{\ell}\right), B^{\ell}\left(\mathbf{x}, \mathbf{x}^{\ell-1}\right)\right) \\
& \leq \mathrm{d}_{\mathrm{TV}}\left(B^{\ell}\left(\mathbf{x}, \mathbf{x}^{\ell}\right), B^{\ell}\left(\mathbf{x}^{\ell}, \mathbf{x}\right)\right) \\
& +\mathrm{d}_{\mathrm{TV}}\left(B^{\ell}\left(\mathbf{x}^{\ell}, \mathbf{x}\right), B^{\ell}\left(\mathbf{x}^{\ell-1}, \mathbf{x}\right)\right) \\
& +\mathrm{d}_{\mathrm{TV}}\left(B^{\ell}\left(\mathbf{x}^{\ell-1}, \mathbf{x}\right), B^{\ell}\left(\mathbf{x}, \mathbf{x}^{\ell-1}\right)\right) \\
& \leq 0+\varepsilon+0=\varepsilon .
\end{aligned}
$$

Using the observations above, for every $\ell \in[n]$ we have

$$
\begin{aligned}
& \underset{\mathbf{x}, \mathbf{x}^{\prime}, \mathcal{W}}{\mathbb{E}}\left[B^{\ell}\left(\mathbf{x}, \mathbf{x}^{\ell}\right)-B^{\ell}\left(\mathbf{x}, \mathbf{x}^{\ell-1}\right)\right] \\
& \leq 2 \Delta \cdot \mathrm{d}_{\mathrm{TV}}\left(B^{\ell}\left(\mathbf{x}, \mathbf{x}^{\ell}\right), B^{\ell}\left(\mathbf{x}, \mathbf{x}^{\ell-1}\right)\right) \\
& \leq 2 \Delta \varepsilon .
\end{aligned}
$$

Thus we have the desired upper bound on the expectation of $B^{\ell}\left(\mathbf{x}, \mathbf{x}^{\ell}\right)-B^{\ell}\left(\mathbf{x}, \mathbf{x}^{\ell-1}\right)$. The corresponding lower bound follows from an analogous argument. This completes the proof.

\subsubsection{From De-Correlated Expectation to Accuracy on Average}

THEOREM 4.7. Let $Q_{\Delta}$ be the family of $\Delta$-sensitive queries on $\mathcal{X}$. Assume that $\mathcal{M}$ is

1. $(\varepsilon=\alpha / 4 \Delta n)-T V$ stable for $k$ adaptively chosen queries from $Q=Q_{\Delta}$ and

2. $\left(\alpha^{\prime}=\alpha / 2\right)$-accurate on average with respect to its sample for $n$ samples from $\mathcal{X}$ for $k$ adaptively chosen queries from $Q$.

Then $\mathcal{M}$ is $\alpha$-accurate on average with respect to the population for $k$ adaptively chosen queries from $Q$ given $n$ samples from $\mathcal{X}$.

The high level approach of the proof is to apply the Lemma 4.6 to a "monitoring algorithm" that watches the interaction between the mechanism $\mathcal{M}(\mathbf{x})$ and the analyst $\mathcal{A}$ and then outputs the least accurate query. Since $\mathcal{M}(\mathbf{x})$ is stable, the de-correlated expectation lemma says that the query output by the monitor will satisfy $q(\mathbf{P}) \approx q(\mathbf{x})$ in expectation, this implies that even for the least accurate query in the interaction between $\mathcal{M}(\mathbf{x})$ and $\mathcal{A}, q(\mathbf{P}) \approx q(\mathbf{x})$ in expectation Thus, if $\mathcal{M}$ is accurate with respect to the sample $\mathbf{x}$, it is also accurate with respect to $\mathbf{P}$

Proof of Theorem 4.7 Let $\mathcal{M}$ be an interactive mechanism and $\mathcal{A}$ be an analyst that chooses the distribution

$$
\mathcal{W}(\mathbf{x})=\mathcal{W}_{\mathbf{P}}[\mathcal{M}, \mathcal{A}](\mathbf{x}):
$$

Input: $\mathrm{x} \in \mathcal{X}^{n}$

Simulate $\mathcal{M}(\mathbf{x})$ and $\mathcal{A}$ interacting, let $q_{1}, \ldots, q_{k} \in$ $Q$ be the queries of $\mathcal{A}$ and let $a_{1}, \ldots, a_{k} \in \mathbb{R}$ be the corresponding answers of $\mathcal{M}$.

Let $j=\arg \max _{j=1, \ldots, k}\left|\operatorname{err}^{\mathbf{P}}\left(q_{j}, a_{j}\right)\right|$.

If $a_{j}-q_{j}(\mathbf{P}) \geq 0$, let $q^{*}=q_{j}$, otherwise let $q^{*}=$ $-q_{j} .\left(Q_{\Delta}\right.$ is closed under negation.)

Output: $q^{*}$.

$\mathbf{P}$. We define the following monitoring algorithm. If $\mathcal{M}$ is stable then so is $\mathcal{W}$, and this fact follows easily from the post-processing lemma (Lemma 2.4).

Claim 4.8. For every $\varepsilon \geq 0$, if the mechanism $\mathcal{M}$ is $\varepsilon$ $T V$ stable for $k$ adaptively chosen queries from $Q$, then for every $\mathbf{P}$ and $\mathcal{A}$, the monitor $\mathcal{W}_{\mathbf{P}}[\mathcal{M}, \mathcal{A}]$ is $\varepsilon-T V$ stable.

Proof of Claim 4.8. The assumption that $\mathcal{M}$ is $\varepsilon$-TV stable for $k$ adaptively chosen queries from $Q$ means that for every analyst $\mathcal{A}$ who asks $k$ queries from $Q$, the algorithm $\mathcal{W}^{\prime}(\mathbf{x})$ that simulates the interaction between $\mathcal{M}(\mathbf{x})$ and $\mathcal{A}$ and outputs the resulting query-answer pairs is $\varepsilon$-TV stable. Observe that the algorithm $\mathcal{W}$ defined above is simply a post-processing of these query-answer pairs. That is, $q^{*}$ depends only on $q_{1}, a_{1}, \ldots, q_{k}, a_{k}$ and $\mathbf{P}$, and not on $\mathbf{x}$. Thus, by Lemma 2.4 for every $\mathbf{P}$ and $\mathcal{A}$, the monitor $\mathcal{W}_{\mathbf{P}}[\mathcal{M}, \mathcal{A}]$ is $\varepsilon$-TV stable.

In light of Claim 4.8 and our assumption that $\mathcal{M}$ is $(\alpha / 4 \Delta n)$ TV stable, we can apply Lemma 4.6 to obtain

$$
\left|\underset{\mathbf{x}, \mathcal{W}}{\mathbb{E}}\left[q^{*}(\mathbf{P})-q^{*}(\mathbf{x}) \mid q^{*}=\mathcal{W}(\mathbf{x})\right]\right| \leq 2\left(\frac{\alpha}{4 \Delta n}\right) \Delta n \leq \alpha / 2 .
$$

To complete the proof, we show that if $\mathcal{M}$ is not $\alpha$-accurate on average with respect to the population $\mathbf{P}$, then $\sqrt{5}$ cannot hold.

Claim 4.9. If $\mathcal{M}$ is $(\alpha / 2)$-accurate for the sample but not $\alpha$-accurate for the population, then

$$
\left|\underset{\mathbf{x}, \mathcal{W}}{\mathbb{E}}\left[q^{*}(\mathbf{P})-q^{*}(\mathbf{x}) \mid q^{*}=\mathcal{W}(\mathbf{x})\right]\right| \geq \alpha / 2 .
$$

Proof of Claim 4.9. Using our assumptions, we can calculate as follows.

$$
\begin{aligned}
& \left|\underset{\mathbf{x}, \mathcal{W}}{\mathbb{E}}\left[q^{*}(\mathbf{P})-q^{*}(\mathbf{x}) \mid q^{*}=\mathcal{W}(\mathbf{x})\right]\right| \\
& =\left|\begin{array}{r}
\underset{\mathbf{x}, \mathcal{W}}{\mathbb{E}}\left[q^{*}(\mathbf{P})-a_{q^{*}} \mid q^{*}=\mathcal{W}(\mathbf{x})\right] \\
+\underset{\mathbf{x}, \mathcal{W}}{\mathbb{E}}\left[a_{q^{*}}-q^{*}(\mathbf{x}) \mid q^{*}=\mathcal{W}(\mathbf{x})\right]
\end{array}\right| \\
& \geq\left|\underset{\mathbf{x}, \mathcal{W}}{\mathbb{E}}\left[q^{*}(\mathbf{P})-a_{q^{*}} \mid q^{*}=\mathcal{W}(\mathbf{x})\right]\right| \\
& -\left|\underset{\mathbf{x}, \mathcal{W}}{\mathbb{E}}\left[a_{q^{*}}-q^{*}(\mathbf{x}) \mid q^{*}=\mathcal{W}(\mathbf{x})\right]\right| \\
& >\alpha-\left|\underset{\mathbf{x}, \mathcal{W}}{\mathbb{E}}\left[a_{q^{*}}-q^{*}(\mathbf{x}) \mid q^{*}=\mathcal{W}(\mathbf{x})\right]\right| \\
& \geq \alpha-\alpha / 2=\alpha / 2 \text {. }
\end{aligned}
$$


Line (6) follows from two observations. First, by construction of $\mathcal{W}$, we always have $q^{*}(\mathbf{P})-a_{q^{*}} \leq 0$. Second, since $\mathcal{M}$ is assumed not to be $\alpha$-accurate on average for the population, the expected value of $\left|q^{*}(\mathbf{P})-a_{q^{*}}\right|>\alpha$. Since $\mathcal{W}$ ensures that $a_{q^{*}}-q^{*}(\mathbf{P}) \geq 0$, we also have that the absolute value of the expectation of $q^{*}(\mathbf{P})-a_{q^{*}}$ is greater than $\alpha$. Line (7) follows from the assumption that $\mathcal{M}$ is $(\alpha / 2)$ accurate on average for the sample.

Thus, if $\mathcal{M}$ is not $\alpha$-accurate on average for the population, we will obtain a contradiction to (5). This completes the proof.

\section{FROM LOW-SENSITIVITY QUERIES TO OPTIMIZATION QUERIES}

In this section, we extend our results for low-sensitivity queries to the more general family of minimization queries. To do so, we design a suitable monitoring algorithm for minimization queries. As in our analysis of low-sensitivity queries, we will have the monitoring algorithm take as input many independent samples and simulate the interaction between $\mathcal{M}$ and $\mathcal{A}$ on each of those samples. Thus, if $\mathcal{M}$ has even a small probability of being inaccurate, then with constant probability the monitor will find a minimization query that $\mathcal{M}$ has answered inaccurately. Previously, we had monitor simply output this query and applied Lemma 3.2 to arrive at a contradiction. However, since Lemma 3.2 only applies to algorithms that output a low-sensitivity query, we can't apply it to the monitor that outputs a minimization query. We address this by having the monitor output the error function associated with the loss function and answer it selects, which is a low-sensitivity query. If we assume that the mechanism is accurate for its sample but not for the population, then the monitor will find a loss function and an answer with low error on the sample but large error on the population. Thus the error function will be a lowsensitivity query with very different answers on the sample and the population, which is a contradiction. To summarize, we have the following theorem.

Theorem 5.1 (Transfer Theorem for Minimization QUERIES). Let $Q=Q_{\min }$ be the family of $\Delta$-sensitive minimization queries on $\mathcal{X}$. Assume that, for some $\alpha, \beta \geq 0, \mathcal{M}$ is

1. $(\varepsilon=\alpha / 128 \Delta n, \delta=\alpha \beta / 64 \Delta n)$-max-KL stable for $k$ adaptively chosen queries from $Q$ and

2. $\left(\alpha^{\prime}=\alpha / 8, \beta^{\prime}=\alpha \beta / 32 \Delta n\right)$-accurate with respect to its sample for $n$ samples from $\mathcal{X}$ for $k$ adaptively chosen queries from $Q$.

Then $\mathcal{M}$ is $(\alpha, \beta)$-accurate with respect to the population for $k$ adaptively chosen queries from $Q$ given $n$ samples from $\mathcal{X}$.

The formal proof is nearly identical to that of Theorem 3.3 . so we omit the full proof. Instead, we will simply describe the modified monitoring algorithm.

\section{APPLICATIONS}

\subsection{Low-Sensitivity and Statistical Queries}

We now plug known stable mechanisms (designed in the context of differential privacy) in to Theorem 3.3 to obtain mechanisms that provide strong error guarantees with high probability for both low-sensitivity and statistical queries.

$$
\mathcal{W}(\mathbf{X})=\mathcal{W}_{\mathbf{P}}[\mathcal{M}, \mathcal{A}](\mathbf{X}):
$$

Input: $\mathbf{X}=\left(\mathbf{x}_{1}, \ldots, \mathbf{x}_{T}\right) \in\left(\mathcal{X}^{n}\right)^{T}$

For $t=1, \ldots, T$ :

Simulate $\mathcal{M}\left(\mathbf{x}_{t}\right)$ and $\mathcal{A}$ interacting, let $L_{t, 1}, \ldots, L_{t, k} \in Q$ be the queries of $\mathcal{A}$ and let $\theta_{t, 1}, \ldots, \theta_{t, k} \in \mathbb{R}$ be the corresponding answers of $\mathcal{M}$.

Let $\left(t^{*}, j^{*}\right)$ be

$$
\left(t^{*}, j^{*}\right)=\underset{j \in[k], t \in[T]}{\arg \max }\left|\operatorname{err}^{\mathbf{P}}\left(L_{t, j}, \theta_{t, j}\right)\right| .
$$

Let $q^{*}(\mathbf{x})=\operatorname{err}_{\mathbf{x}}\left(L_{t^{*}, j^{*}}, \theta_{t^{*}, j^{*}}\right)$ (note, by construction, $q^{*} \in Q_{2 \Delta}$, i.e. $q^{*}$ is $2 \Delta$-sensitive)

Output: $\left(q^{*}, t^{*}\right)$.

Corollary 6.1 (Theorem 3.3 And SU15A]). There is a mechanism $\mathcal{M}$ that is $(\alpha, \beta)$-accurate with respect to the population for $k$ adaptively chosen queries from $Q_{\Delta}$ where $\Delta=O(1 / n)$ given $n$ samples from $\mathcal{X}$ for

$$
n \geq O\left(\frac{\sqrt{k \cdot \log \log k} \cdot \log ^{3 / 2}(1 / \alpha \beta)}{\alpha^{2}}\right)
$$

The mechanism runs in time $\operatorname{poly}(n, \log |\mathcal{X}|, \log (1 / \beta))$ per query.

Corollary 6.2 (Theorem 3.3 And [RR10]). There is a mechanism $\mathcal{M}$ that is $(\alpha, \beta)$-accurate with respect to the population for $k$ adaptively chosen queries from $Q_{\Delta}$ where $\Delta=O(1 / n)$ given $n$ samples from $\mathcal{X}$ for

$$
n=O\left(\frac{\log |\mathcal{X}| \cdot \log k \cdot \log ^{3 / 2}(1 / \alpha \beta)}{\alpha^{3}}\right)
$$

The mechanism runs in time poly $\left(|\mathcal{X}|^{n}\right)$ per query. The case where $\Delta$ is not $O(1 / n)$ can be handled by rescaling the output of the query.

Corollary 6.3 (Theorem 3.3 AND HR10]). There is a mechanism $\mathcal{M}$ that is $\alpha$-accurate on average with respect to the population for $k$ adaptively chosen queries from $Q_{S Q}$ given $n$ samples from $\mathcal{X}$ for

$$
n=O\left(\frac{\sqrt{\log |\mathcal{X}|} \cdot \log k \cdot \log ^{3 / 2}(1 / \alpha \beta)}{\alpha^{3}}\right)
$$

The mechanism runs in time $\operatorname{poly}(n,|\mathcal{X}|)$ per query.

\subsection{Optimization Queries}

The results of the Section 5 can be combined with existing differentially private algorithms for minimizing "empirical risk" (that is, loss with respect to the sample $x$ ) to obtain algorithms for answering adaptive sequences of minimization queries. We provide a few specific instantiations here, based on known differentially private mechanisms.

\subsubsection{Minimization Over Arbitrary Finite Sets}

Corollary 6.4 (Theorem 5.1 And MT07). Let $\Theta$ be a finite set of size at most $D$. Let $Q \subset Q_{\text {min }}$ be the set of sensitivity- $1 / n$ loss functions bounded between 0 and $C$. 
Then there is a mechanism $\mathcal{M}$ that is $(\alpha, \beta)$-accurate with respect to the population for $k$ adaptively chosen queries from $Q_{\min }$ given

$$
n \geq O\left(\frac{\log (D C / \alpha) \cdot \sqrt{k} \cdot \log ^{3 / 2}(1 / \alpha \beta)}{\alpha^{2}}\right)
$$

samples from $\mathcal{X}$. The running time of the mechanism is dominated by $O((k+\log (1 / \beta)) \cdot D)$ evaluations of the loss function.

\subsubsection{Convex Minimization}

We state bounds for convex minimization queries for some of the most common parameter regimes in applications. In the first two corollaries, we consider 1-Lipschit $2^{6}$ loss functions over a bounded domain.

Corollary 6.5 (Theorem 5.1 And BST14]). Let $\Theta$ be a closed, convex subset of $\mathbb{R}^{d}$ set such that $\max _{\theta \in \Theta}\|\theta\|_{2} \leq 1$. Let $Q \subset Q_{\text {min }}$ be the set of convex 1-Lipschitz loss functions that are $1 / n$-sensitive. Then there is a mechanism $\mathcal{M}$ that is $(\alpha, \beta)$-accurate with respect to the population for $k$ adaptively chosen queries from $Q$ given

$$
n=\tilde{O}\left(\frac{\sqrt{d k} \cdot \log ^{2}(1 / \alpha \beta)}{\alpha^{2}}\right)
$$

samples from $Q$. The running time of the mechanism is dominated by $k \cdot n^{2}$ evaluations of the gradient $\nabla L$.

Corollary 6.6 (TheOREM 5.1 AND Ull15). Let $\Theta$ be a closed, convex subset of $\mathbb{R}^{d}$ set such that $\max _{\theta \in \Theta}\|\theta\|_{2} \leq 1$. Let $Q \subset Q_{\min }$ be the set of convex 1-Lipschitz loss functions that are $1 / n$-sensitive. Then there is a mechanism $\mathcal{M}$ that is $(\alpha, \beta)$-accurate with respect to the population for $k$ adaptively chosen queries from $Q$ given

$$
n=\tilde{O}\left(\frac{\sqrt{\log |\mathcal{X}|} \cdot(\sqrt{d}+\log k) \cdot \log ^{3 / 2}(1 / \alpha \beta)}{\alpha^{3}}\right)
$$

samples from $\mathcal{X}$. The running time of the mechanism is dominated by $\operatorname{poly}(n,|\mathcal{X}|)$ and $k \cdot n^{2}$ evaluations of the gradient $\nabla L$.

In the next two corollaries, we consider 1-strongly convex $7^{7}$ Lipschitz loss functions over a bounded domain.

Corollary 6.7 (Theorem 5.1 and BST14]). Let $\Theta$ be a closed, convex subset of $\mathbb{R}^{d}$ set such that $\max _{\theta \in \Theta}\|\theta\|_{2} \leq 1$. Let $Q \subset Q_{\text {min }}$ be the set of 1-strongly convex, 1-Lipschitz loss functions that are $1 / n$-sensitive. Then there is a mechanism $\mathcal{M}$ that is $(\alpha, \beta)$-accurate with respect to the population for $k$ adaptively chosen queries from $Q$ given

$$
n=\tilde{O}\left(\frac{\sqrt{d k} \cdot \log ^{3 / 2}(1 / \alpha \beta)}{\alpha^{3 / 2}}\right)
$$

samples from $\mathcal{X}$. The running time of the mechanism is dominated by $k \cdot n^{2}$ evaluations of the gradient $\nabla L$.

${ }^{6}$ A loss function $L: \mathcal{X} \times \mathbb{R}^{d} \rightarrow \mathbb{R}$ is 1-Lipschitz if for every $\theta, \theta^{\prime} \in \mathbb{R}^{d}, x \in \mathcal{X},\left|L(\theta, x)-L\left(\theta^{\prime}, x\right)\right| \leq\left\|\theta-\theta^{\prime}\right\|_{2}$.

${ }^{7} \mathrm{~A}$ loss function $L: \mathcal{X} \times \mathbb{R}^{d} \rightarrow \mathbb{R}$ is 1 -strongly convex if for every $\theta, \theta^{\prime} \in \mathbb{R}^{d}, x \in \mathcal{X}$,

$$
L\left(\theta^{\prime}, x\right) \geq L(\theta, x)+\left\langle\nabla L(\theta, x), \theta^{\prime}-\theta\right\rangle+(1 / 2) \cdot\left\|\theta-\theta^{\prime}\right\|_{2}^{2},
$$

where the (sub)gradient $\nabla L(\theta, x)$ is taken with respect to $\theta$.
Corollary 6.8 (Theorem 5.1 And Ull15). Let $\Theta$ be a closed, convex subset of $\mathbb{R}^{d}$ set such that $\max _{\theta \in \Theta}\|\theta\|_{2} \leq 1$. Let $Q \subset Q_{\min }$ be the set of 1-strongly convex 1-Lipschitz loss functions that are $1 / n$-sensitive. Then there is a mechanism $\mathcal{M}$ that is $(\alpha, \beta)$-accurate with respect to the population for $k$ adaptively chosen queries from $Q$ given

$$
n=\tilde{O}\left(\sqrt{\log |\mathcal{X}|} \cdot\left(\frac{\sqrt{d}}{\alpha^{5 / 2}}+\frac{\log k}{\alpha^{3}}\right) \cdot \log ^{3 / 2}(1 / \alpha \beta)\right)
$$

samples from $\mathcal{X}$. The running time of the mechanism is dominated by $\operatorname{poly}(n,|\mathcal{X}|)$ and $k \cdot n^{2}$ evaluations of the gradient $\nabla L$.

\section{ALTERNATIVE FORM OF GENERALIZA- TION AND TIGHTNESS OF RESULTS}

We now provide an alternative form of our generalization bounds. The following Theorem is more general than Theorem 3.3 because it says that no max-KL stable procedure that outputs a low-sensitivity can output a query that distinguishes the sample from the population (not just max-KL stable procedures that are accurate for the sample).

First we prove the following technical lemma.

Lemma 7.1. Let $F$ be a finite set, $f: F \rightarrow \mathbb{R}$ a function, and $\eta>0$. Define a random variable $X$ on $F$ by

$$
\mathbb{P}[X=x]=\frac{e^{\eta f(x)}}{C}, \quad \text { where } \quad C=\sum_{x \in F} e^{\eta f(x)} .
$$

Then

$$
\mathbb{E}[f(X)] \geq \max _{x \in F} f(x)-\frac{1}{\eta} \log |F| .
$$

Proof. We have

$$
f(x)=\frac{1}{\eta}(\log C+\log \mathbb{P}[X=x]) .
$$

Thus

$$
\begin{aligned}
\mathbb{E}[f(X)] & =\sum_{x \in F} \mathbb{P}[X=x] f(x) \\
& =\sum_{x \in F} \mathbb{P}[X=x] \frac{1}{\eta}(\log C+\log \mathbb{P}[X=x]) \\
& =\frac{1}{\eta}(\log C-H(X)),
\end{aligned}
$$

where $H(X)$ is the Shannon entropy of the distribution of $X$ (measured in nats, rather than bits). In particular,

$$
H(X) \leq \log |\operatorname{support}(X)|=\log |F|,
$$

as the uniform distribution maximizes entropy. Moreover, $C \geq \max _{x \in F} e^{\eta f(x)}$, whence $\frac{1}{\eta} \log C \geq \max _{x \in F} f(x)$. The result now follows from these two inequalities.

Theorem 7.2. Let $\varepsilon \in(0,1 / 3), \delta \in(0, \varepsilon / 4)$, and $n \geq$ $\frac{1}{\varepsilon^{2}} \log \left(\frac{4 \varepsilon}{\delta}\right) . \quad$ Let $\mathcal{M}: \mathcal{X}^{n} \rightarrow Q_{\Delta}$ be $(\varepsilon, \delta)$-max-KL stable where $Q_{\Delta}$ is the class of $\Delta$-sensitive queries $q: \mathcal{X}^{n} \rightarrow \mathbb{R}$. Let $\mathbf{P}$ be a distribution on $\mathcal{X}$, let $\mathbf{x} \leftarrow_{R} \mathbf{P}^{n}$, and let $q \leftarrow_{R} \mathcal{M}(\mathbf{x})$. Then

$$
\underset{\mathbf{x}, \mathcal{M}}{\mathbb{P}}[|q(\mathbf{P})-q(\mathbf{x})| \geq 18 \varepsilon \Delta n]<\frac{\delta}{\varepsilon}
$$


Intuitively, Theorem 7.2 says that "stability prevents overfitting." It says that no stable algorithm can output a lowsensitivity function that distinghishes its input from the population the input was drawn from (i.e. "overfits" its sample).

In particular, Theorem 7.2 implies that, if a mechanism $\mathcal{M}$ is stable and outputs $q$ that "fits" its data, then $q$ also "fits" the population. This gives a learning theory perspective on our results.

Proof. Consider the following monitor algorithm $\mathcal{W}$.

$$
\mathcal{W}(\mathbf{X})=\mathcal{W}_{\mathbf{P}}[\mathcal{M}](\mathbf{X}):
$$

Input: $\mathbf{X}=\left(\mathbf{x}_{1}, \ldots, \mathbf{x}_{T}\right) \in\left(\mathcal{X}^{n}\right)^{T}$

Set $F=\emptyset$.

For $t=1, \ldots, T$ :

Let $q_{t} \leftarrow \mathcal{M}\left(\mathbf{x}_{t}\right)$, and set $F=F \cup$ $\left\{\left(q_{t}, t\right),\left(-q_{t}, t\right)\right\}$.

Sample $\left(q^{*}, t^{*}\right)$ from $F$ with probability proportional to $\exp \left(\frac{\varepsilon}{\Delta}\left(q^{*}\left(\mathbf{x}_{t^{*}}\right)-q^{*}(\mathbf{P})\right)\right)$.

Output: $\left(q^{*}, t^{*}\right)$.

We will use the monitor $\mathcal{W}$ with $T=\lfloor\epsilon / \delta\rfloor$. Observe that $\mathcal{W}$ only access its input through $\mathcal{M}$ (which is $(\varepsilon, \delta)$-max-KLstable) and the exponential mechanism (which is $(\varepsilon, 0)$-maxKL-stable). Thus, by composition and postprocessing, $\mathcal{W}$ is $(2 \varepsilon, \delta)$-max-KL stable. We can hence apply Lemma 3.2 to obtain

$$
\begin{array}{r}
\underset{\mathbf{X}, \mathcal{W}}{\mathbb{E}}\left[q^{*}\left(\mathbf{x}_{t^{*}}\right)-q^{*}(\mathbf{P}) \mid\left(q^{*}, t^{*}\right)=\mathcal{W}(\mathbf{X})\right] \\
\leq 2\left(e^{2 \varepsilon}-1+T \delta\right) \Delta n<8 \varepsilon \Delta n
\end{array}
$$

Now we can apply Lemma 7.1 with $f(q, t)=q\left(\mathbf{x}_{t}\right)-q(\mathbf{P})$ and $\eta=\frac{\varepsilon}{\Delta}$ to get

$$
\begin{array}{r}
\underset{q^{*}, t^{*}}{\mathbb{E}}\left[f\left(q^{*}, t^{*}\right)\right] \geq \max _{(q, t) \in F} f(q, t)-\frac{\Delta}{\varepsilon} \log |F| \\
=\max _{t \in[T]}\left|q_{t}\left(\mathbf{x}_{t}\right)-q_{t}(\mathbf{P})\right|-\frac{\Delta}{\varepsilon} \log (2 T) .
\end{array}
$$

Combining (8) and (9) gives

$$
\begin{array}{r}
\underset{\mathbf{X}, \mathcal{W}}{\mathbb{E}}\left[\max _{t \in[T]}\left|q_{t}\left(\mathbf{x}_{t}\right)-q_{t}(\mathbf{P})\right|\right]-\frac{\Delta}{\varepsilon} \log (2 T) \\
\leq \underset{\mathbf{X}, \mathcal{W}}{\mathbb{E}}\left[q^{*}\left(\mathbf{x}_{t^{*}}\right)-q^{*}(\mathbf{P}) \mid\left(q^{*}, t^{*}\right)=\mathcal{W}(\mathbf{X})\right]<8 \varepsilon \Delta n .
\end{array}
$$

To complete the proof, we assume, for the sake of contradition, that $\mathcal{M}$ has a high enough probability of outputting a query $q$ such that $|q(\mathbf{P})-q(\mathbf{x})|$ is large. To obtain a contradiction from this assumption, we need the following natural claim (analogous to Claim 3.5 about the output of the monitor.

Claim 7.3. If

$$
\underset{\mathbf{x}, \mathcal{M}}{\mathbb{P}}[|q(\mathbf{P})-q(\mathbf{x})| \geq 18 \varepsilon \Delta n] \geq \frac{\delta}{\varepsilon},
$$

then

$\underset{\mathbf{X}, \mathcal{W}}{\mathbb{P}}\left[\max _{t \in[T]}\left|q_{t}\left(\mathbf{x}_{t}\right)-q_{t}(\mathbf{P})\right| \geq 18 \varepsilon \Delta n\right] \geq 1-\left(1-\frac{\delta}{\varepsilon}\right)^{T} \geq \frac{1}{2}$.
Thus

$$
\underset{\mathbf{X}, \mathcal{W}}{\mathbb{E}}\left[\max _{t \in[T]}\left|q_{t}\left(\mathbf{x}_{t}\right)-q_{t}(\mathbf{P})\right|\right] \geq 9 \varepsilon \Delta n
$$

Combining (10) and 11 gives

$$
9 \varepsilon \Delta n-\frac{\Delta}{\varepsilon} \log (2 T) \leq 8 \varepsilon \Delta n,
$$

which simiplies to

$$
\log (2 \varepsilon / \delta) \geq \log (2 T) \geq \varepsilon^{2} n .
$$

This contradicts the assumption that $n \geq \frac{1}{\varepsilon^{2}} \log \left(\frac{4 \varepsilon}{\delta}\right)$ and hence completes the proof.

\subsection{Optimality}

We now show that our connection between max-KL stability and generalization (Theorem 7.2 and Theorem 3.3 is optimal.

LEMma 7.4. Let $\alpha>\delta>0$, let $n \geq \frac{1}{\alpha}$, and let $\Delta \in[0,1]$. Let $\mathcal{U}$ be the uniform distribution over $[0,1]$. There exists a $(0, \delta)$-max-KL stable algorithm $\mathcal{A}:[0,1]^{n} \rightarrow Q_{\Delta}$ such that if $\mathbf{X} \leftarrow_{R} \mathcal{U}^{n}$ and if $q \leftarrow_{R} \mathcal{A}(\mathbf{X})$ then

$$
\operatorname{Pr}[q(\mathbf{X})-q(\mathcal{U}) \geq \alpha \Delta n] \geq \frac{\delta}{2 \alpha} .
$$

Proof. Consider the following simple algorithm, denoted as $\mathcal{B}$. On input a database $\mathbf{x}$, output $\mathbf{x}$ with probability $\delta$, and otherwise output the empty database. Clearly, $\mathcal{B}$ is $(0, \delta)$-max-KL stable. Now construct the following algorithm $\mathcal{A}$.

Input: $\mathrm{A}$ database $\mathbf{X} \in[0,1]^{n}$. We think of $\mathbf{X}$ as $\frac{1}{\alpha}$ databases of size $\alpha n$ each: $\mathbf{X}=\left(\mathbf{x}_{1}, \ldots, \mathbf{x}_{1 / \alpha}\right)$.

For $1 \leq i \leq 1 / \alpha$ let $\hat{\mathbf{x}}_{i}=\mathcal{B}\left(\mathbf{x}_{i}\right)$.

Let $p:[0,1] \rightarrow\{0,1\}$ where $p(x)=1$ iff $\exists i$ s.t. $x \in \hat{\mathbf{x}}_{i}$.

Define $q_{p}:[0,1]^{n} \rightarrow \mathbb{R}$ where $q_{p}(\mathbf{x})=\Delta \sum_{x \in \mathbf{x}} p(x)$ (note that $q_{p}$ is a $\Delta$-sensitive query, and that it is a statistical query if $\Delta=1 / n)$.

Output: $q_{p}$.

As $\mathcal{B}$ is $(0, \delta)$-max-KL stable, and as $\mathcal{A}$ only applies $\mathcal{B}$ on disjoint databases, we get that $\mathcal{A}$ is also $(0, \delta)$-max-KL stable.

Suppose $\mathbf{X}=\left(\mathbf{x}_{1}, \ldots, \mathbf{x}_{1 / \alpha}\right)$ contains i.i.d. samples from $\mathcal{U}$, and consider the execution of $\mathcal{A}$ on $\mathbf{X}$. Observe that the predicate $p$ evaluates to 1 only on a finite number of points from $[0,1]$, and hence, we have that $q_{p}(\mathcal{U})=0$. Next note that $q_{p}(\mathbf{X})=\alpha \Delta n \cdot\left|\left\{i: \hat{\mathbf{x}}_{i}=\mathbf{x}_{i}\right\}\right|$. Therefore, if there exists an $i$ s.t. $\hat{\mathbf{x}}_{i}=\mathbf{x}_{i}$ then $q_{p}(\mathbf{X})-q_{p}(\mathcal{U}) \geq \alpha \Delta n$. The probability that this is not the case is at most

$$
(1-\delta)^{1 / \alpha} \leq e^{-\delta / \alpha} \leq 1-\frac{\delta}{2 \alpha}
$$

ans thus, with probability at least $\frac{\delta}{2 \alpha}$, algorithm $\mathcal{A}$ outputs a $\Delta$-sensitive query $q_{p}$ s.t. $q_{p}(\mathbf{X})-q_{p}(\mathcal{U}) \geq \alpha \Delta n$.

In particular, using Lemma 7.4 with $\alpha=\varepsilon$ shows that the parameters in Theorem 7.2 are tight. 


\section{ACKNOWLEDGEMENTS}

We thank Mark Bun, Moritz Hardt, Aaron Roth, and Salil Vadhan for many helpful discussions.

\section{REFERENCES}

[BE02] Olivier Bousquet and André Elisseeff. Stability and generalization. JMLR, 2:499-526, 2002.

[BH95] Yoav Benjamini and Yosef Hochberg. Controlling the false discovery rate: a practical and powerful approach to multiple testing. Journal of the Royal Statistical Society. Series B (Methodological), 57(1):289-300, 1995.

[BH15] Avrim Blum and Moritz Hardt. The ladder: A reliable leaderboard for machine learning competitions. CoRR, abs/1502.04585, 2015.

[Bon36] Carlo Emilio Bonferroni. Teoria statistica delle classi e calcolo delle probabilita. Pubbl. $d$. R. Ist. Super. di Sci. Econom. e Commerciali di Firenze., 8, 1936.

[BSSU15] Raef Bassily, Adam Smith, Thomas Steinke, and Jonathan Ullman. More general queries and less generalization error in adaptive data analysis. CoRR, abs/1503.04843, 2015.

[BST14] Raef Bassily, Adam Smith, and Abhradeep Thakurta. Private empirical risk minimization: Efficient algorithms and tight error bounds. In FOCS, pages 464-473, 2014.

[BUV14] Mark Bun, Jonathan Ullman, and Salil P. Vadhan. Fingerprinting codes and the price of approximate differential privacy. In $S T O C$, pages 1-10. ACM, May 31 - June 32014.

$\left[\mathrm{DFH}^{+} 15 \mathrm{a}\right]$ Cynthia Dwork, Vitaly Feldman, Moritz Hardt, Toniann Pitassi, Omer Reingold, and Aaron Roth. Generalization in adaptive data analysis and holdout reuse. In NIPS, 2015.

$\left[\mathrm{DFH}^{+} 15 \mathrm{~b}\right]$ Cynthia Dwork, Vitaly Feldman, Moritz Hardt, Toniann Pitassi, Omer Reingold, and Aaron Roth. Preserving statistical validity in adaptive data analysis. In STOC, 2015.

$\left[\mathrm{DFH}^{+} 15 \mathrm{c}\right]$ Cynthia Dwork, Vitaly Feldman, Moritz Hardt, Toniann Pitassi, Omer Reingold, and Aaron Roth. The reusable holdout: Preserving validity in adaptive data analysis. Science, 349(6248):636-638, June 2015.

[DMNS06] Cynthia Dwork, Frank McSherry, Kobbi Nissim, and Adam Smith. Calibrating noise to sensitivity in private data analysis. In $T C C$, pages 265-284. Springer, March 4-7 2006.

[DN03] Irit Dinur and Kobbi Nissim. Revealing information while preserving privacy. In PODS, pages 202-210. ACM, June 9-12 2003.

[DRV10] Cynthia Dwork, Guy N. Rothblum, and Salil P. Vadhan. Boosting and differential privacy. In FOCS, pages 51-60, 2010.

[Dun61] Olive Jean Dunn. Multiple comparisons among means. JASA, 56:52-64, 1961.

[DW79a] Luc Devroye and Terry J. Wagner. Distribution-free inequalities for the deleted and holdout error estimates. IEEE Transactions on Information Theory, 25(2):202-207, 1979.
[DW79b] Luc Devroye and Terry J. Wagner. Distribution-free performance bounds for potential function rules. IEEE Transactions on Information Theory, 25(5):601-604, 1979.

[Dwo06] Cynthia Dwork. Differential privacy. In ICALP, pages 1-12, 2006.

[GL14] Andrew Gelman and Eric Loken. The statistical crisis in science. American Scientist, 102(6):460, 2014.

[HR10] Moritz Hardt and Guy Rothblum. A multiplicative weights mechanism for privacy-preserving data analysis. In FOCS, pages 61-70. IEEE, 2010.

[HU14] Moritz Hardt and Jonathan Ullman. Preventing false discovery in interactive data analysis is hard. In FOCS, 2014.

[Ioa05] John P. A. Ioannidis. Why most published research findings are false. PLoS Medicine, 2(8):124, August 2005.

[Kea93] Michael J. Kearns. Efficient noise-tolerant learning from statistical queries. In $S T O C$, pages 392-401. ACM, May 16-18 1993.

[KR99] Michael J. Kearns and Dana Ron. Algorithmic stability and sanity-check bounds for leave-one-out cross-validation. Neural Computation, 11(6):1427-1453, 1999.

[McS] Frank McSherry. Differential privacy for measure concentration. Blog post. February 4 , 2014. http://windowsontheory.org/

[MT07] Frank McSherry and Kunal Talwar. Mechanism design via differential privacy. In FOCS, pages 94-103. IEEE, Oct 20-23 2007.

[NS15] Kobbi Nissim and Uri Stemmer. On the generalization properties of differential privacy. CoRR, abs/1504.05800, 2015.

[RR10] Aaron Roth and Tim Roughgarden. Interactive privacy via the median mechanism. In $S T O C$, pages 765-774. ACM, June 5-8 2010.

[RZ16] Daniel Russo and James Zou. Controlling bias in adaptive data analysis using information theory. In AISTATS, 2016.

[SSSS10] Shai Shalev-Shwartz, Ohad Shamir, Nathan Srebro, and Karthik Sridharan. Learnability, stability and uniform convergence. JMLR, 11:2635-2670, 2010.

[SU15a] Thomas Steinke and Jonathan Ullman. Between pure and approximate differential privacy. CoRR, abs/1501.06095, 2015.

[SU15b] Thomas Steinke and Jonathan Ullman. Interactive fingerprinting codes and the hardness of preventing false discovery. In COLT, pages 1588-1628, 2015.

[Ull13] Jonathan Ullman. Answering $\mathrm{n}^{2+o(1)}$ counting queries with differential privacy is hard. In STOC, pages 361-370. ACM, June 1-4 2013.

[Ull15] Jonathan Ullman. Private multiplicative weights beyond linear queries. In $P O D S$. ACM, May 31-June 42015.

[WLF16] Yu-Xiang Wang, Jing Lei, and Stephen E. Fienberg. A minimax theory for adaptive data analysis. arXiv:1602.04287 [stat.ML], 2016. 